\title{
The AGN contribution to mid-infrared surveys
}

\section{X-ray counterparts of the mid-IR sources in the Lockman Hole and HDF- $N^{\star}$}

\author{
D. Fadda ${ }^{1,2}$, H. Flores ${ }^{2,3}$, G. Hasinger ${ }^{4}$, A. Franceschini ${ }^{5}$, B. Altieri ${ }^{6}$, C. J. Cesarsky ${ }^{7}$, \\ D. Elbaz ${ }^{2}$, and Ph. Ferrando ${ }^{2}$ \\ 1 Instituto de Astrofísica de Canarias (IAC), Via Lactea S/N, 38205 La Laguna, Tenerife, Spain \\ 2 CEA Saclay - Service d'Astrophysique, Orme des Merisiers, 91191 Gif-sur-Yvette Cedex, France \\ 3 Observatoire de Paris Meudon, DAEC, 92195 Meudon Principal Cedex, France \\ 4 AIP, An der Sternwarte 16, 14482 Potsdam, Germany \\ 5 Dipartimento di Astronomia, Università di Padova, Vicolo dell'Osservatorio 5, 35122 Padova, Italy \\ 6 XMM-Newton Operations Centre, ESA Vilspa, Apartado 50727, 28080 Madrid, Spain \\ 7 ESO, Karl-Schwarzschild Straße 2, 85748 Garching bei München, Germany
}

Received 12 June 2001 / Accepted 20 December 2001

\begin{abstract}
We provide constraints on the AGN contribution to the mid-IR extragalactic background light from a correlation analysis of deep X-ray and mid-IR observations in two regions centred on the Lockman Hole and Hubble Deep Field North (HDF-N). The Lockman region, of more than 200 square arcminutes, was observed by ISOCAM and XMM-Newton to a depth of $0.3 \mathrm{mJy}$ at $15 \mu \mathrm{m}$ (resolving more than $30 \%$ of the mid-IR background). In the same area XMM-Newton reached flux limits of $1.4 \times 10^{-15} \mathrm{erg} \mathrm{cm}^{-2} \mathrm{~s}^{-1}$ in the $2-10 \mathrm{keV}$ energy band and $2.4 \times 10^{-15} \mathrm{erg} \mathrm{cm}^{-2} \mathrm{~s}^{-1}$ in the $5-10 \mathrm{keV}$ energy band, resolving about $80 \%$ of the $2-10 \mathrm{keV}$ and $60 \%$ of the 5-10 keV backgrounds (the deepest observation in this hard band to date). Among the 76 galaxies detected by XMM-Newton, 24 show mid-IR emission, but the relative percentage of X-ray sources with mid-IR counterparts increases with the band energy: from $30 \%$ of the $0.5-2 \mathrm{keV}$ sources up to $63 \%$ of the $5-10 \mathrm{keV}$ sources. In contrast, only a small fraction of the mid-IR sources (around 10\%) show X-ray emission within the sensitivity limits of XMM-Newton observations. The region centred on the HDF-N has been observed by ISOCAM (24 square arcminutes) to a depth of $0.05 \mathrm{mJy}$ (more than $60 \%$ of the mid-IR background is resolved at this limit) and covered with a $1 \mathrm{Msec}$ exposure by Chandra. In this case, $25 \%$ of the mid-IR sources are detected in the X-ray, while $30-40 \%$ of the X-ray sources show mid-IR emission. Under the assumption that all XMM sources except stars or galaxy clusters are AGN-dominated, AGNs contribute $(15 \pm 5) \%$ of the total mid-IR flux in the Lockman Hole. For the HDF-N we have assumed that AGN-dominated sources are luminous X-ray sources and sources with SEDs from radio to X-ray wavelengths typical of local AGNs, in which case we find that $(18 \pm 7) \%$ of the mid-IR flux are due to AGN emission. If we put together all the existing information from the deepest HDF-N data to the bright large-area sample in the ELAIS S1 region observed with BeppoSAX (for a total of 50 X-ray-mid-IR matched sources) using the median mid-IR to X-ray spectral indices as a function of the X-ray flux, we find an AGN contribution to the $15 \mu \mathrm{m}$ background of $(17 \pm 2) \%$. Since the IR spectra of typical AGNs peak around $20 \mu \mathrm{m}$ while starburst spectra peak at significantly longer infrared wavelengths, this figure may be considered as an upper limit to the AGN contribution to the CIRB energy density. We conclude that the population of IR luminous galaxies detected in the ISOCAM deep surveys, and the CIRB sources themselves, are mainly constituted by dust-obscured starbursts.
\end{abstract}

Key words. cosmology: observations - infrared: galaxies - X-ray: galaxies: quasars: general

\section{Introduction}

Send offprint requests to: D. Fadda,

e-mail: fadda@ll.iac.es

* Partially based on observations with ISO and XMM, ESA projects with instruments funded by ESA Member States with the participation of ISAS and NASA, and on observations with the Canada-France-Hawaii Telescope at Mauna Kea, Hawaii.
The discovery of luminous mid-IR sources in the deep ISOCAM surveys (Aussel et al. 1999; Elbaz et al. 1999) sheds new light on the star formation history of the Universe (Madau et al. 1996; Rowan-Robinson et al. 1997; Flores et al. 1999) and the origin of the infrared extragalactic background light (Puget et al. 1998; 
Elbaz et al. 2002). Mid-IR observations appear to be fundamental to our understanding of the evolution of the Universe because they offer views of obscured star formation with a resolution sufficient to identify optical counterparts of the infrared emitters, which is often not the case of far-IR and sub-mm observations. In particular, the ISOCAM $15 \mu \mathrm{m}$-centred band has proven to be well suited to the study of star formation up to redshifts of 1.5 (Elbaz et al. 2002), as well as to the resolution of a large part of the infrared background detected by the DIRBE and FIRAS experiments (Puget et al. 1998; Fixsen et al. 1998; Hauser et al. 1998). Since a large part of the star formation is detectable only in the infrared and ISOCAM sources are rare with respect to optical/UV sources, this implies that a large part of star forming activity occurs in rare, very luminous systems.

Obscured AGNs are known to be the major contributors to the hard X-ray background (e.g. Comastri et al. 1995) and a large fraction of their optical-UV energy is re-radiated at longer wavelengths, from the near-infrared to the sub-millimetre region. Although the shape of the infrared spectrum of such sources is unknown, some authors claim that absorbed AGNs could contribute a substantial fraction (up to 50\%) of the infrared background (Almaini et al. 1999; Fabian \& Iwasawa 1999). However, the nature of the energy source in powerful infrared emitters is still a matter of debate. Recent ISO results suggest that most of these galaxies could host an AGN, that but their emission is dominated by vigorous star formation (Genzel et al. 1998; Lutz et al. 1998). Tran et al. (2001), analysing almost 80 mid-IR spectra of luminous and ultraluminous infrared galaxies, found that the average contributions of star formation to the infrared luminosity are $82-94 \%$ for low-luminosity sources $\left(L_{\mathrm{IR}}<10^{12.4} L_{\odot}\right)$ and $44-55 \%$ for high luminosity sources $\left(L_{\mathrm{IR}} \geq 10^{12.4} L_{\odot}\right)$.

If the bulk of the ISOCAM $15 \mu \mathrm{m}$ galaxies, which have a typical luminosity in the $10^{11} L_{\odot}<L_{\mathrm{IR}}<10^{12} L_{\odot}$ range and make up most of the infrared background (Elbaz et al. 2002), were dusty starbursts, this would have important implications on the star formation history of the Universe (see Flores et al. 1999).

When detailed optical spectra are not available, it is in principle possible to classify mid-IR galaxies using mid-IR based diagnostic diagrams (Laurent et al. 2000) or fitting templates with multi-wavelength data (Flores et al. 1999). The major drawback of the multi-wavelength approach is the similarity of starburst and Seyfert-2 SEDs in the radio-optical domain (see examples in Flores et al. 1999). On the other hand, the mid-IR diagnostic by Laurent et al. (2000), which is based on only five local AGNs, strongly depends on the source redshift. Moreover, in the case of deep surveys, large photometric errors make it difficult to distinguish between starburst- and AGN-dominated midIR sources.

A direct approach consists of cross-correlating mid-IR and X-ray observations to detect AGNs and, in particular, using hard X-ray energy bands which are less sensitive to the extinction and are thus able to reveal obscured AGNs.
In this perspective, we compared a recent XMMNewton observation (Hasinger et al. 2001) with the midIR ISOCAM surveys at $6.75 \mu \mathrm{m}$ and $15 \mu \mathrm{m}$ (Fadda et al. 2002) of a region located in the Lockman Hole and the deep Chandra observation (Brandt et al. 2001a) with the ISOCAM survey (Aussel et al. 1999) of a region centred on the Hubble Deep Field.

Pioneering studies of this type have been already tried by comparing BeppoSAX with ISOCAM data in the Elais-S1 field (Alexander et al. 2001) and Chandra with ISOCAM data in the HDF-N field (Hornschemeier et al. 2001). However, these studies are respectively affected by the low sensitivity of the surveys and the small area observed. The present one is the first study with a sufficient depth and sky coverage to obtain a statistically significant set of sources with hard X-ray and mid-IR emission.

In this paper, after discussing the cross-correlation of mid-IR and X-ray catalogues and the common optical, mid-IR and X-ray properties of the sources, we compare the sources detected in the surveys made in the Lockman, HDF-N and Elais-S1 regions with ISOCAM and a variety of X-ray satellites (XMM, Chandra and Beppo-SAX) with local templates. Finally, we estimate the AGN contribution to the extragalactic infrared light.

\section{Observations}

A region of $20 \times 20$ square arcminutes in the Lockman Hole, centred on the sky position 10:52:07+57:21:02 (J2000), which corresponds to the centre of the ROSAT HRI image (Hasinger et al. 1993, 1998), has been surveyed by ISOCAM, the mid-IR camera on board ISO (Fadda et al. 2002). The field was observed for a total of $45 \mathrm{ks}$ at $15 \mu \mathrm{m}$ and $70 \mathrm{ks}$ at $6.75 \mu \mathrm{m}$. Moreover, a shallow survey has been done at $15 \mu \mathrm{m}$ on a region of $40 \times 40 \operatorname{arcmin}^{2}$ with the same centre for a total exposure time of $55 \mathrm{ks}$. Combining the two surveys, ISOCAM observed at $15 \mu \mathrm{m}$ the central region for a total of $60 \mathrm{ks}$.

If we compare this observation with the deepest ISOCAM surveys performed in the HDF-N region (24.3 square arcminutes for $22 \mathrm{ks}$ at $15 \mu \mathrm{m}$ and 10.4 square arcminutes for $23 \mathrm{ks}$ at $6.75 \mu \mathrm{m}$, Aussel et al. 1999), the Lockman Deep Survey is 16 times more extended and $\sim 4$ times shallower at $15 \mu \mathrm{m}$ than the HDF-N survey (the sensitivity depending on the integration time and on the redundancy of the observations). ISOCAM data have been reduced using the PRETI pipeline (see Starck et al. 1999) with a few improvements as described by Fadda et al. (2000).

The XMM-Newton observation of the Lockman Hole has been done during the verification phase of the satellite (Hasinger et al. 2001) and is centred on the sky position 10:52:43+57:28:48 (J2000) which was the centre of the PSPC ROSAT image (Hasinger et al. 1998). The total exposure time of this observation was $190 \mathrm{ks}$, but only $100 \mathrm{ks}$ are usable because of bad space weather (solar activity) during the observations. The limiting fluxes of these 

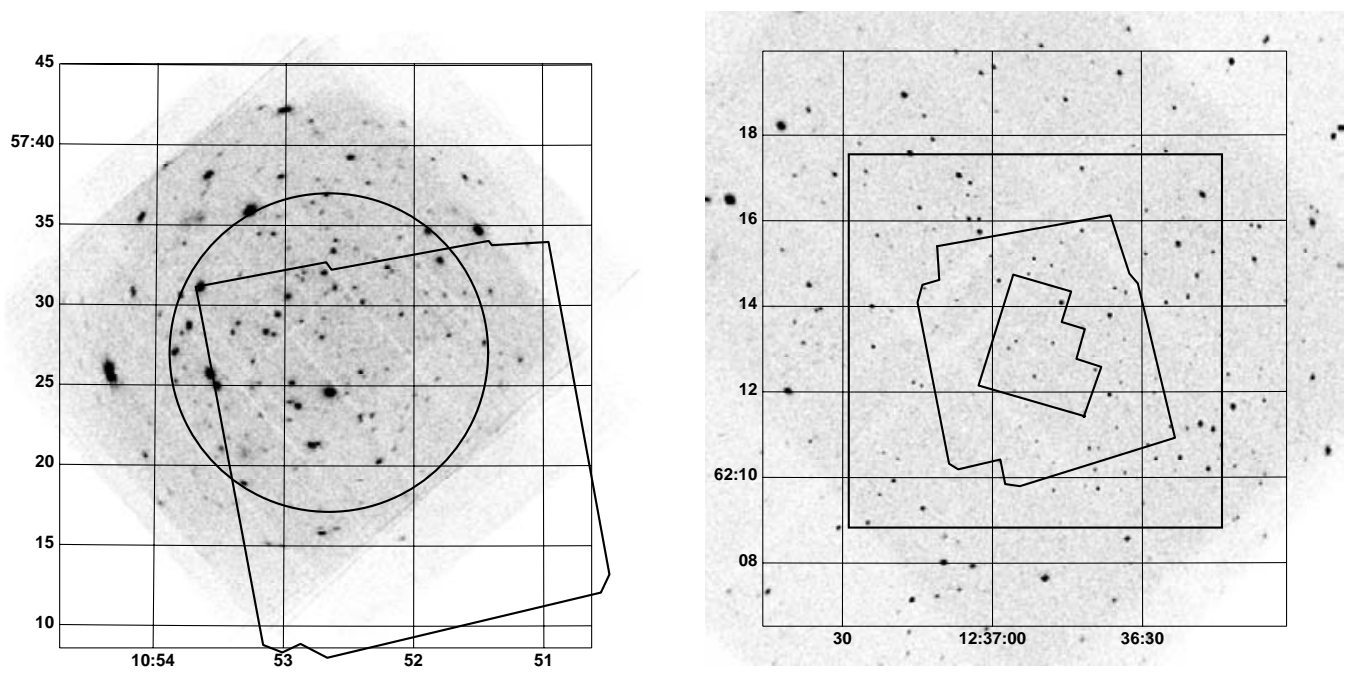

Fig. 1. Relative positions of the mid-IR and X-ray surveys. On the left, on the full-band (0.5-10 keV) XMM image of the Lockman Hole we superimpose the contours of the area deeply surveyed by ISOCAM and the circle from inside which Hasinger et al. (2001) sources have been extracted. The area considered in the paper results from the intersection of these two regions. On the right, on the full-band $(0.5-8 \mathrm{keV})$ Chandra image of the Hubble Deep Field and flanking fields we draw: the field observed with the Hubble Space Telescope, the field surveyed with ISOCAM (irregular contour) and the Caltech area (square).

observations are $3 \times 10^{-16} \mathrm{erg} \mathrm{cm}^{-2} \mathrm{~s}^{-1}$ in the $0.5-2 \mathrm{keV}$ band and $1.4 \times 10^{-15} \mathrm{erg} \mathrm{cm}^{-2} \mathrm{~s}^{-1}$ in the $2-10 \mathrm{keV}$ band.

To match X-ray and mid-IR sources we considered Xray sources detected at the $4 \sigma$ level inside an off-axis angle of 10 arcmin (Hasinger et al. 2001).

Due to the different centre of the ISOCAM and XMMNewton observations, the size of the overlapping region is 218 square arcminutes (see Fig. 1), which corresponds to $70 \%$ of the XMM-Newton region. In this region, a total of 76 sources was detected in the various X-ray bands excluding clusters and stars $(68,42$ and 19 in the $0.5-2 \mathrm{keV}$, 2-10 keV and 5-10 keV energy bands, respectively). In the same area, 184 and 65 extragalactic sources were detected by ISOCAM at the $3 \sigma$ level in the $L W 3$ band and at $4 \sigma$ level in the $L W 2$ band which have central wavelenghts of $15 \mu \mathrm{m}$ and $6.75 \mu \mathrm{m}$, respectively (Fadda et al. 2002). As shown in Fig. 1, the region centred on the Hubble Deep Field observed by ISOCAM (Rowan-Robinson et al. 1997) has been completely covered by deep $1 \mathrm{Ms}$ Chandra observations (Brandt et al. 2001a). The Chandra observations are one order of magnitude deeper than the XMMNewton observations in the $0.5-2 \mathrm{keV}$ band (flux limit of $3 \times 10^{-17} \mathrm{erg} \mathrm{cm}^{-2} \mathrm{~s}^{-1}$ ) and $2-8 \mathrm{keV}$ band (flux limit of $\left.2 \times 10^{-16} \mathrm{erg} \mathrm{cm}^{-2} \mathrm{~s}^{-1}\right)$. On the contrary, Chandra is less sensitive than XMM-Newton in the ultra-hard band $(>5 \mathrm{keV})$. In the $\mathrm{X}$-ray- $L W 3$ common area, Chandra detects a total of 59 sources in the full X-ray band $(0.5-$ $8 \mathrm{keV}$ ) and 50, 40 and 23 sources in the $0.5-2 \mathrm{keV}, 2-8 \mathrm{keV}$ and 4-8 keV bands, respectively. Aussel et al. (1999) list a total of $93 L W 3$ sources, 42 of which have a flux greater than $0.1 \mathrm{mJy}$ (completeness flux limit of the survey, Aussel et al. 2002). In the X-ray- $L W 2$ common area (10.4 square arcminutes with 10 ISOCAM detections), Chandra detects a total of 24 sources in the full band and 22, 12
Table 1. Number of X-ray sources detected at $15 \mu \mathrm{m}$ and $6.75 \mu \mathrm{m}$ in the Lockman Hole-centred region and percentage with one-sigma errors of common detections relative to the total number of X-ray and mid-IR sources in the different XMMNewton bands. Poissonian errors are computed according to Gehrels et al. (1986).

\begin{tabular}{ccccccc}
\hline XMM & \multicolumn{3}{c}{$L W 3$} & \multicolumn{3}{c}{$L W 2$} \\
band & $\#$ & $\mathrm{X} \%$ & $\mathrm{IR} \%$ & $\#$ & $\mathrm{X} \%$ & $\mathrm{IR} \%$ \\
\hline Soft & 20 & $29_{-10}^{+12}$ & $11_{-3}^{+3}$ & 6 & $9_{-5}^{+6}$ & $9_{-5}^{+7}$ \\
Hard & 16 & $38_{-15}^{+19}$ & $9_{-4}^{+6}$ & 5 & $12_{-7}^{+10}$ & $8_{-4}^{+6}$ \\
U-hard & 12 & $63_{-32}^{+42}$ & $7_{-2}^{+3}$ & 5 & $26_{-17}^{+25}$ & $8_{-4}^{+6}$ \\
Full & 22 & $29_{-9}^{+11}$ & $12_{-5}^{+7}$ & 7 & $9_{-4}^{+6}$ & $11_{-5}^{+7}$ \\
\hline
\end{tabular}

Table 2. Number of X-ray sources detected at $15 \mu \mathrm{m}$ and $6.75 \mu \mathrm{m}$ in the Hubble Deep Field-centred region and percentage with one-sigma errors of common detections relative to the total number of X-ray and mid-IR sources in the different Chandra bands. Poissonian errors are computed according to Gehrels et al. (1986).

\begin{tabular}{ccccccc}
\hline Chandra & \multicolumn{3}{c}{$L W 3$} & \multicolumn{4}{c}{$L W 2$} \\
band & $\#$ & $\mathrm{X} \%$ & $\mathrm{IR} \%$ & $\#$ & $\mathrm{X} \%$ & $\mathrm{IR} \%$ \\
\hline Soft & 20 & $40_{-15}^{+18}$ & $21_{-7}^{+8}$ & 5 & $23_{-15}^{+21}$ & $50_{-37}^{+55}$ \\
Hard & 12 & $30_{-13}^{+17}$ & $13_{-5}^{+6}$ & 2 & $17_{-6}^{+10}$ & $20_{-19}^{+35}$ \\
U-hard & 7 & $30_{-18}^{+24}$ & $8_{-4}^{+5}$ & 2 & $29_{-4}^{+6}$ & $20_{-19}^{+35}$ \\
Full & 22 & $37_{-13}^{+15}$ & $24_{-7}^{+9}$ & 5 & $21_{-13}^{+19}$ & $50_{-37}^{+55}$ \\
\hline
\end{tabular}

and 7 sources in the soft, hard and ultra-hard bands, respectively.

Tables 1 and 2 summarise the percentages of X-ray and mid-IR sources which emit in the mid-IR and X-ray bands, respectively. It appears clear that a large fraction 


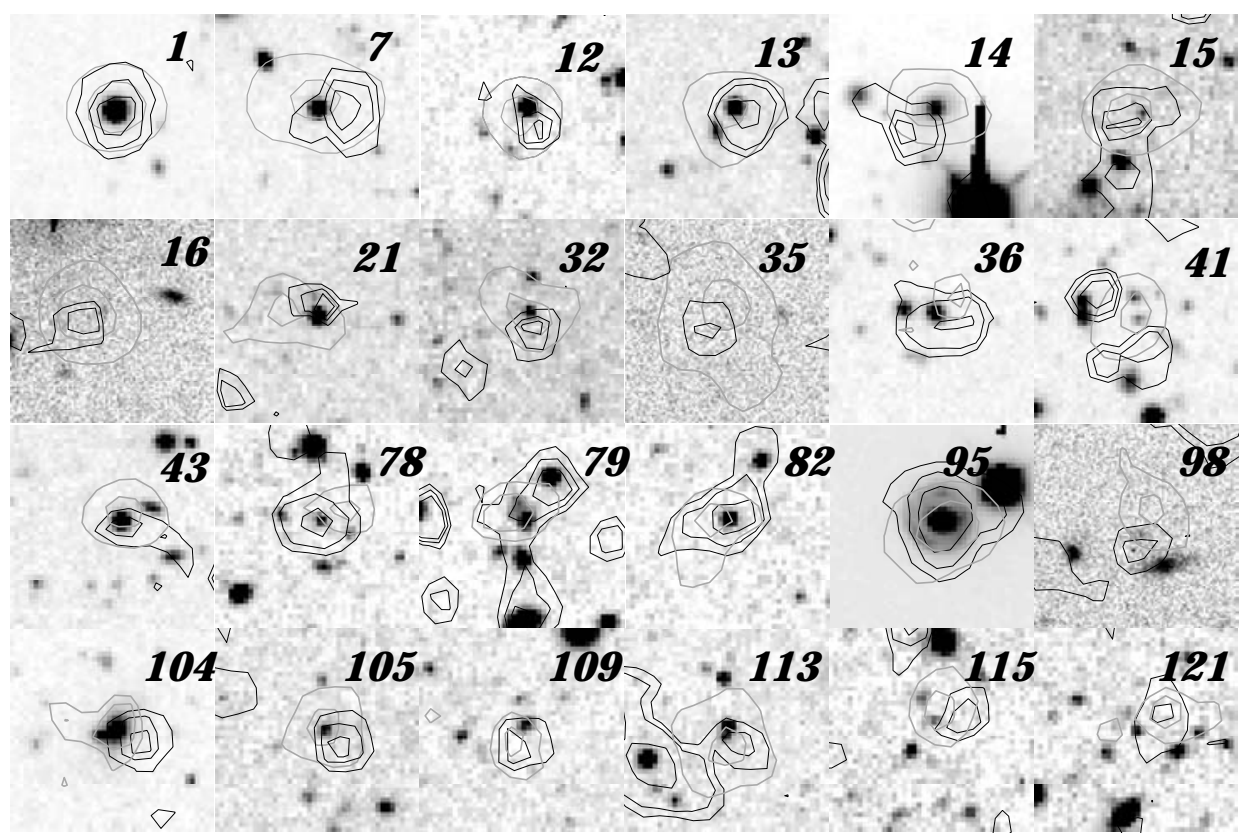

Fig. 2. Finding charts of the common X-ray and mid-IR sources in the Lockman Hole region (see Table 3). X-ray and mid-IR isocontours are plotted on optical images ( $I$-band) with grey and black lines, respectively. X-ray contours come from the $0.5-$ $7 \mathrm{keV}$ image, while mid-IR contours refer to the $15 \mu \mathrm{m}$ image, except for sources \#104 and \#121 which have been detected only in the $6.75 \mu \mathrm{m}$ image. The size of each image is $25^{\prime \prime} \times 25^{\prime \prime}$.

of X-ray sources have an $L W 3$ counterpart. In particular, in the case of the Lockman Hole, the percentage of X-ray sources emitting in the $5-10 \mathrm{keV}$ band with $L W 3$ counterpart is greater than $60 \%$. The same does not occur in the case of the Chandra deep field, probably because the 4-8 keV ultra-hard band of Chandra is not so sensitive as the similar band of XMM-Newton. On the other hand, only around $10 \%$ of the $L W 3$ sources are detected in the various X-ray bands except for the soft X-ray band in the HDF-N, where the extremely deep Chandra observations are able also to detect normal galaxies.

In the case of $L W 2$ observations, we have less detections with respect to $L W 3$ and a similar trend of detections as a function of the energy band in the Lockman Hole. The case of the Hubble Deep Field is not very constraining because of the bad quality of the $L W 2$ observations (only 10 extragalactic sources have been detected).

\section{Basic analysis}

\subsection{Cross-correlation of $X$-ray and mid-IR catalogues}

In the case of the Lockman Hole observations, XMM sources have position errors of 1-3" (Hasinger et al. 2001), while $15 \mu \mathrm{m}$ and $6.75 \mu \mathrm{m}$ ISOCAM sources have position errors of $2-4^{\prime \prime}$ (depending on the redundancy of mid-IR observation, see Fadda et al. 2002). In practice, we match $\mathrm{X}$-ray and mid-IR sources within a circle of $4^{\prime \prime}$. In total, we found 22 matches with $15 \mu \mathrm{m}$ sources detected at the $3 \sigma$ level and 7 with $6.75 \mu \mathrm{m}$ sources at the $4 \sigma$ level. For the sake of completeness, we list also upper limits of the $L W 2$ and $L W 3$ fluxes when a source is detected in only one of the two ISOCAM filters.
In the case of the Hubble Deep Field image, we have reprocessed the ISOCAM data to recompute more accurate positions of the $L W 3$ sources. We have used the $I$-band image of Barger et al. (1999) available on the $\mathrm{Web}^{1}$ to which we added the astrometry according to the catalogue of Hogg et al. (2000). Position errors of the Chandra sources are estimated to be less than $1^{\prime \prime}$ (Brandt et al. 2001a), while ISOCAM sources thanks to the microscan technique of observation have errors less than $2^{\prime \prime}$. Therefore, we match X-ray and mid-IR sources within a circle of $2^{\prime \prime}$.

For each source we have computed the probability of random association of the X-ray source with its mid-IR and optical counterparts, and of the mid-IR source with its optical counterpart. Assuming that the counterpart belongs to a Poissonian distributed population of sources,

$$
P=1-\mathrm{e}^{-n(A) \pi d^{2}}
$$

gives the probability to have a random association within a distance $d$ (distance between the source and the possible counterpart) with a source brighter than $A$ (the flux of the possible counterpart). $n(A)$ is the expected number of sources with flux (magnitude) greater (lower) than that of the possible counterpart $A$. It has been evaluated using the distribution of mid-IR, X-ray fluxes, and the counts in selected regions of the $I$-band image whitout bright stars.

Contours of the matched sources are also plotted on optical images in Figs. 2 and 3. In most of the cases there is a clear correspondence between X-ray and mid-IR sources. Only in a few cases (\#149 in the Hubble Deep

\footnotetext{
1 www.ifa.hawaii.edu/ cowie/hdflank/hdflank.html
} 

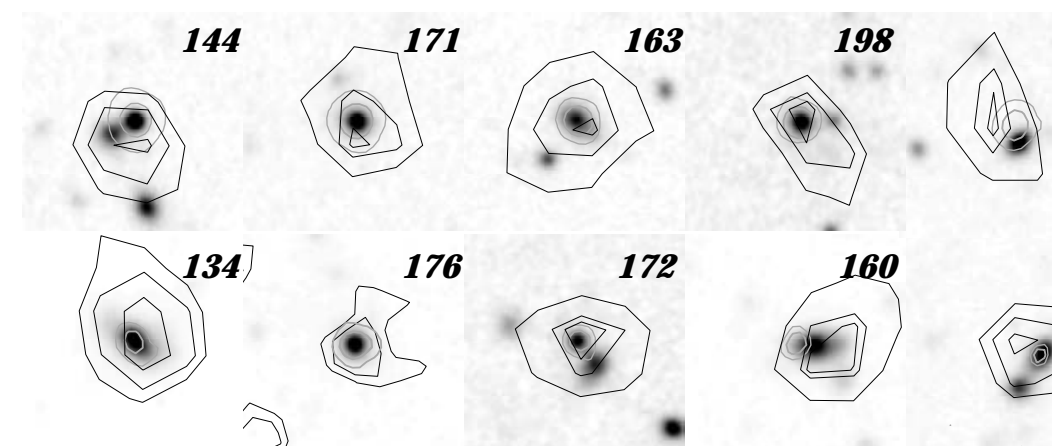

190

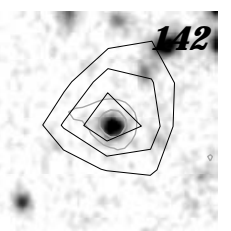

176
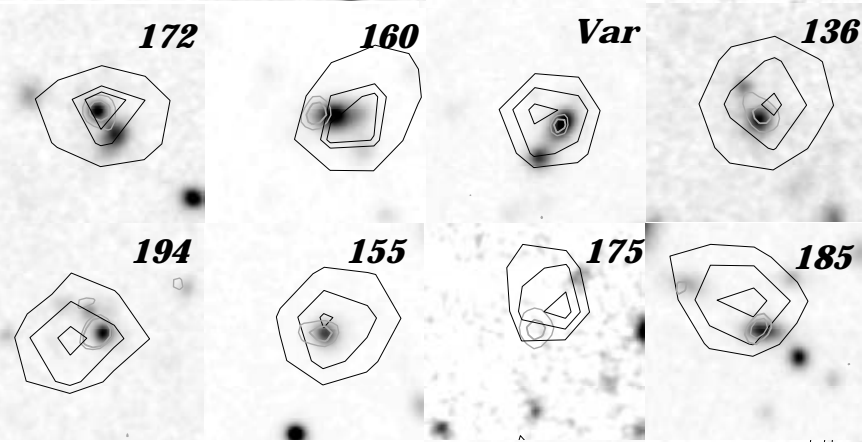

161

188
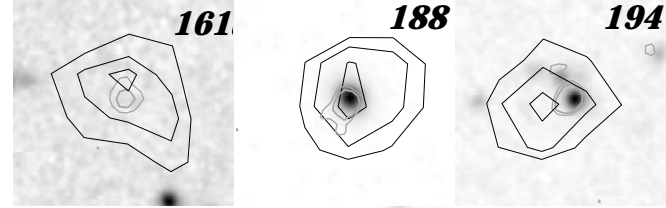

178
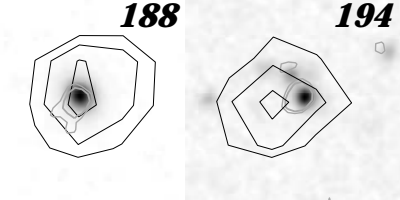

220

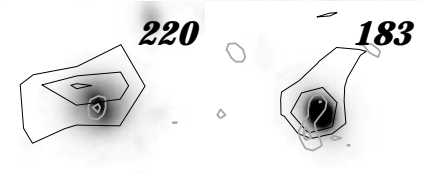

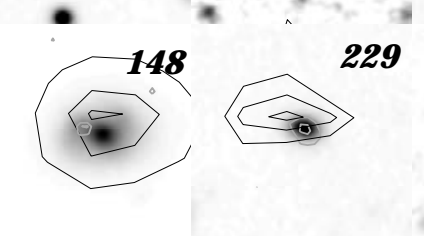
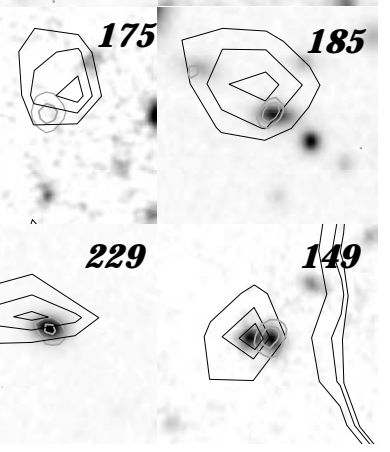

Fig. 3. Finding charts of the common X-ray and mid-IR sources in the Hubble Deep Field and Flanking Fields (see Table 4). $\mathrm{X}$-ray and mid-IR isocontours are plotted on optical images ( $I$-band, Barger et al. 1999) with grey and black lines, respectively. X-ray contours come from the $2-8 \mathrm{keV}$ Chandra image, while mid-IR contours refer to the $15 \mu \mathrm{m}$ image except for sources \# 176 and \#183 which have been detected only in the $6.75 \mu \mathrm{m}$ image. The size of each image is $15^{\prime \prime} \times 15^{\prime \prime}$. Sources \#161 and \#175 correspond to blank fields in the $I$-image.

Field and \#41, \#79 in the Lockman Hole) optical counterparts are uncertain and the match relies only on the distance criterion. Three sources are optically faint (see Alexander et al. 2001a).

Results of these cross-correlations are reported in Tables 3 and 4 which contain positions of the X-ray sources, distances, and probability of random associations between sources and proposed counterparts, optical magnitudes in the $V, I, R$ and $K$ bands, X-ray and midIR fluxes, redshifts and AGN types when known, as well as other quantities described in the following.

For the sources without spectroscopic redshifts, we have estimated photometric redshifts using four optical magnitudes $(V, I, R$ and $K)$ under the assumption that the optical emission is dominated by the host galaxy. We used a library of synthetic SEDs generated with PEGASE2.0 (Fioc \& Rocca-Volmerange 1997) to fit the distribution of optical magnitudes. The median error on photometric redshifts, derived from a study on the Hubble Deep Field South (Franceschini et al., in prep.), is 0.1.

\subsection{Comparison of mid-IR and X-ray common surveys}

Up to now, only two ISOCAM surveys have been studied in the X-ray bands: the Elais-S1 field (Alexander et al. 2001) and the HDF-N field (Hornschemeier et al. 2001). In this paper we extend the study of mid-IR-X-ray crosscorrelation in the HDF-N field and flanking fields using the new observations of Brandt et al. (2001a) and justifying the associations between X-ray and mid-IR sources.

Figure 4 compares the mid-IR and X-ray fluxes of the sources detected in these surveys as well as the sensitivity limits of the X-ray observations and the $80 \%$ completeness limits of the $15 \mu \mathrm{m}$ surveys. The survey in the Lockman Hole region is intermediate between the ElaisS1 and HDF-N surveys. It covers an area of 218 square arcminutes that is $\sim 30$ times smaller than the Elais-S1 survey ( $\sim 6000$ square arcminutes) and $\sim 10$ times larger than the HDF-N survey (24 square arcminutes). In terms of sensitivity it is approximately one order of magnitude shallower than the HDF-N in the soft and hard X-ray bands, but it is two orders of magnitudes deeper than the Beppo-SAX observations. Moreover, the XMM-Newton data allow us to explore with a good sensitivity the ultrahard energy band (4.5-10 keV), which has been pioneered by Beppo-SAX and is not well covered by Chandra. As we have already seen, this band is very interesting because more than $60 \%$ of the ultra-hard sources in the Lockman Hole have mid-IR counterparts.

Taking into account the limits in sensitivity, the three surveys are compatible in terms of source density. Within the sensitivity limits of the Lockman Hole observations $\left(F_{15 \mu \mathrm{m}} \geq 0.4 \mathrm{mJy}\right.$ and $F_{0.5-2 \mathrm{keV}}>$ $0.3 \times 10^{-15} \mathrm{erg} \mathrm{cm}^{-2} \mathrm{~s}^{-1}, \quad F_{2-10 \mathrm{keV}}>1.4 \times$ $10^{-15} \mathrm{erg} \mathrm{cm}^{-2} \mathrm{~s}^{-1}$ ) we find 13 and 11 sources in the soft and hard X-ray bands, respectively. Therefore, we expect to detect in the HDF-N within the same flux limits $1.4_{-0.4}^{+0.5}$ 
Table 3. List of the XMM-Newton sources with mid-IR counterparts in the Lockman Hole region ordered with decreasing 0.5$2 \mathrm{keV}$ flux. For each association we report: the XMM number with previous ROSAT name within brackets (1); the coordinates of the X-ray source $(2,3)$; the offset between associated sources in arcseconds and the probability of random association in $10^{-3}$ units for X-ray vs. optical $(4,5)$, mid-IR vs. optical $(6,7)$ and X-ray vs. mid-IR $(8,9)$, respectively; the $V, I, R$ and $K^{\prime}$ magnitudes based on CFHT and Calar Alto observations (10-13); the X-ray fluxes in the soft (0.5-2 keV) and hard (2-10 keV) $\mathrm{X}$-ray bands in $10^{-15} \mathrm{cgs}$ units $(14,15)$; the hardness ratios $(16-18)$; the $2-10 \mathrm{keV}$ luminosity $(19)$; the mid-IR fluxes at $6.75 \mu \mathrm{m}$ (20) and $15 \mu \mathrm{m}$ in mJy (21); the $\alpha_{\mathrm{IX}}$ index (22) described in the text; the measured redshift (23) and the AGN type (24). Zero probability of random association means less than $0.5 \times 10^{-3}$. The hardness ratios, defined as $(H-S) /(H+S)($ where $H$ and $S$ represent hard and soft bands, respectively), compare $0.2-0.5$ vs. $0.5-2 \mathrm{keV}, 0.5-2$ vs. $2-4.5 \mathrm{keV}$ and $2-4.5$ vs. $4.5-10 \mathrm{keV}$, respectively. In three cases a $15 \mu \mathrm{m}$ source has been marginally detected $(S N R<4)$ at $6.75 \mu \mathrm{m}$. We report their $6.75 \mu \mathrm{m}$ fluxes inside brackets. Detections in the 5-10 keV X-ray bands are marked with an asterisk on the $2-10 \mathrm{keV}$ flux. The $6.75 \mu \mathrm{m}$ flux of the source \#95 has been derived by deblending two close sources. Redshifts are taken from Lehmann et al. (2000, 2001) except for \#82 (Fadda et al. 2002). Photometric redshifts computed on the basis of $V, I, R$ and $K^{\prime}$ magnitudes are reported inside brackets.

\begin{tabular}{|c|c|c|c|c|c|c|c|c|c|c|c|c|c|c|c|c|c|c|c|c|c|c|c|}
\hline Names & 00 & Coords & 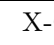 & Op & IR- & $-\mathrm{Op}$ & $\mathrm{X}-1$ & IR & & & pt & & & & & X-ray & & & at & & & & \\
\hline & $10^{\mathrm{h}}$ & $\delta-57^{\circ}$ & & P & $\Delta$ & $P$ & 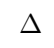 & & V & $V-I$ & $R$ & $-K^{\prime}$ & $S X$ & $H X$ & & ard. R & atio & 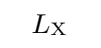 & $W 2$ & $L W 3$ & & $z$ & $T$ \\
\hline (1) & (2) & (3) & (4) & $(5)$ & $(6)$ & $(7)$ & (8) & (9) & $(10)$ & $(11)$ & $(12)$ & (13) & $(14)$ & $(15)$ & 16) & $(17)$ & $(18)$ & 19) & (20) & (21) & 22) & (23) & $(24)$ \\
\hline $1(32 \mathrm{~A})$ & $52: 39.6$ & $24: 32$ & 0.6 & 0 & 0.3 & 0 & 0.1 & 0 & 17.8 & 0.6 & 17.5 & 1.4 & 58.7 & $* 60.2$ & .10 & $0-.71$ & $1-.46$ & 44.61 & $0.9_{-0.3}^{+0.3}$ & & 1.17 & 1.113 & 1 \\
\hline $7(37 \mathrm{~A})$ & $52: 48.3$ & $21: 18$ & 1.5 & 2 & 2.5 & 5 & 2.9 & 2 & 20.3 & 0.9 & 19.9 & 2.6 & 11.2 & 7.7 & -.08 & $8-.79$ & -.44 & 42.80 & $0.3_{-0.3}^{+0.3}$ & $\begin{array}{l}-0.3 \\
-0.3\end{array}$ & .29 & 0.467 & 1 \\
\hline $12(30 \mathrm{~A})$ & 57.2 & $25: 07$ & 1.4 & 3 & 2.6 & 12 & 2.7 & 2 & 21.5 & 1.2 & 20.9 & 2.5 & 6.1 & 7.2 & .16 & $6-.69$ & -.62 & 44.06 & $<0.3$ & $0.6_{-0.4}^{+0.3}$ & .29 & 27 & \\
\hline $13(120 \mathrm{~A})$ & 09.5 & $28: 21$ & 1.0 & 1 & 1.7 & 3 & 2.1 & 1 & 20.7 & 0.9 & 20.4 & 2.0 & & .1 .0 & .20 & $0-.63$ & -.56 & 44.08 & $<0.3$ & $0.8_{-0.2}^{+0.4}$ & & 1 & \\
\hline $14(45 Z)$ & :19.1 & $18: 53$ & 0.5 & 0 & 3.6 & 15 & 4.0 & 5 & 21.3 & 2.3 & 21.2 & - & .7 & 5.2 & .44 & $4-.66$ & -.46 & 43.07 & $<0.3$ & $0.6_{-0.4}^{+0.3}$ & 32 & 0 & 2 \\
\hline $15(14 \mathrm{Z})$ & $52: 42.6$ & $31: 58$ & 2.0 & 96 & 2.1 & 105 & 1.0 & 2 & 25.2 & 1.4 & 25.0 & 5.6 & 4.6 & $* 12.8$ & .96 & $6-.33$ & -.50 & 44.51 & $<0.3$ & $0.7_{-0.2}^{+.35}$ & .26 & 9) & - \\
\hline $16(12 \mathrm{~A})$ & $: 49.6$ & $32: 49$ & 2.5 & 41 & 2.8 & 51 & 0.9 & 0 & 23.2 & - & 22.9 & 4. & & (5) & .93 & $3 \quad .09$ & -.40 & 44.22 & $0.3_{-0.2}^{+0.3}$ & $0.4_{-0.3}^{+0.3}$ & & c & 2 \\
\hline $21(\xi$ & 5 & $21: 23$ & 3.8 & 23 & 1.0 & 2 & 4.0 & 8 & 20.9 & 0.7 & 20.4 & 1.5 & 3 & 2 & .19 & 6 & -.67 & 44.29 & $<0.3$ & $0.3_{-0.2}^{+0.2}$ & 32 & 2 & \\
\hline $32(4$ & $52: 43.4$ & $27: 59$ & 1.0 & 10 & 1.6 & 25 & 0.8 & 0 & 24.7 & 2.2 & 24.4 & 5. & 1.9 & 1.4 & .92 & $2-.41$ & -.39 & 43.36 & $<0.3$ & $0.3_{-0.2}^{+0.3}$ & 1.31 & $(1.0)$ & - \\
\hline 35 & $51: 46.6$ & $30: 35$ & - & - & - & - & 4.0 & 8 & - & - & 25.6 & $4 .($ & 1.8 & - & .64 & $4-.79$ & -.00 & 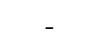 & $<0.3$ & $0.3_{-0.2}^{+0.2}$ & 40 & - & - \\
\hline 36 & & 2 & 3.2 & 18 & 2.3 & 9 & 3.0 & 2 & 21.7 & 1.4 & 22. & & & 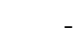 & 22 & 2 & $5-$ & & $<0.3$ & $0.7_{-0.2}^{+0.3}$ & & & 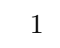 \\
\hline 41 & $51: 28.3$ & $27: 40$ & 0.4 & 1 & 3.7 & 120 & 4.0 & 8 & 24.3 & 1.9 & 22.8 & 3.2 & 1.6 & *12.4 & 1.0 & .14 & $4-.54$ & 44.17 & $<0.3$ & $0.3_{-0.2}^{+0.3}$ & 22 & 1) & \\
\hline 43 & $52: 06.8$ & $29: 26$ & 1.1 & 2 & 0.7 & 1 & 1.9 & 1 & 22.6 & 2.3 & 21.9 & 4. & 1.5 & * 8.4 & .87 & $7-.01$ & -.68 & 43.33 & $<0.3$ & $0.6_{-0.3}^{+0.3}$ & 28 & $(0.75)$ & - \\
\hline 78( & & & 3.0 & 59 & 0.4 & 1 & 3.3 & 0 & 24.6 & 2.6 & 24.2 & & & - & 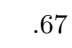 & & - & & $0.4_{-0.3}^{+0.3}$ & $1.4_{-0.3}^{+0.4}$ & & 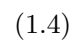 & 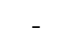 \\
\hline 79 & 0.6 & $: 21$ & 2.1 & 15 & 3.7 & 50 & 4.0 & 6 & 22.4 & 3 & 22. & & 0.7 & $* 2.0$ & .77 & & -.08 & & $<0.3$ & $0.5_{-0.4}^{+0.3}$ & .41 & 0.974 & 1 \\
\hline 82 & 55.0 & $24: 07$ & 2.0 & 12 & 0.4 & 0 & 1.9 & 0 & 22.7 & 1.7 & 22.0 & 3.3 & 0.6 & - & .87 & $7-.27$ & $7-.74$ & $<43.80$ & $<0.3$ & $1.0_{-0.2}^{+0.4}$ & $>1.49$ & 2.4 & - \\
\hline $95(5$ & 52 & 2 & 1.2 & 0 & 0.2 & 0 & 1.3 & 0 & 17.8 & 1.3 & 18.1 & & 0.5 & & .20 & .07 & .37 & 41.60 & $0.7_{-0.3}^{+0.3}$ & $2.4_{-0.5}^{+0.5}$ & 1.50 & 0.204 & 2 \\
\hline 98 & 6.9 & $29: 45$ & 3.0 & 55 & 1.4 & 12 & 4.0 & 8 & 23.0 & 1. & 22. & - & & - & 1.0 & 38 & $3-.77$ & 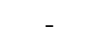 & $<0.3$ & $0.3_{-0.2}^{+0.3}$ & 41 & 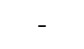 & - \\
\hline 102 & 4.9 & $30: 53$ & 0.1 & 0 & 3.0 & 5 & 3.0 & - & 22.2 & 3.2 & 21.7 & & & - & .36 & $6-.96$ & .84 & $<42.86$ & $0.3_{-0.2}^{+0.3}$ & $<0.7$ & - & $(1.0)$ & 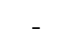 \\
\hline 105 & 53 & $24: 50$ & 0.4 & 2 & 2.6 & 65 & 3.2 & 5 & 25.8 & 3.3 & 24.5 & 5. & 0.4 & 3 & .69 & .26 & -.28 & 43.20 & $<0.3$ & $0.3_{-0.2}^{+0.3}$ & .30 & $(0.8)$ & - \\
\hline 109 & $51: 44.6$ & $26: 51$ & 2.2 & 50 & 2.1 & 46 & 1.6 & 1 & 24.3 & 1.7 & 23.9 & 4. & 0.4 & - & .90 & $0-.64$ & $4-.82$ & $<42.97$ & $<0.3$ & $0.3_{-0.2}^{+0.2}$ & $>1.40$ & (1.1) & - \\
\hline $11:$ & $53: 05.6$ & $28: 10$ & 1.5 & 12 & 1.5 & 12 & 1.3 & 0 & 23.3 & 1.6 & 23.0 & 4 & & 0.0 & - & - .63 & -.13 & 43.55 & $<0.3$ & $0.7_{-0.2}^{+0.4}$ & .30 & $(0.9)$ & - \\
\hline 115 & & $24: 30$ & 1.2 & 36 & 3.3 & 140 & 3.1 & 5 & 26.4 & 2.6 & 24.7 & 5.3 & & $* 4.9$ & - & .35 & -.18 & 43.51 & $<0.3$ & $0.3_{-0.2}^{+0.3}$ & 29 & $(1.1)$ & - \\
\hline 121 & 31.4 & 25:04 & 3.5 & 195 & 3.4 & 205 & 0.5 & - & 25.3 & 1.9 & 24.9 & 5.1 & & * 2.3 & - & $\begin{array}{r}-.51 \\
\end{array}$ & .07 & 43.08 & $0.3_{-0.2}^{+0.3}$ & $<0.5$ & - & $(1.0)$ & - \\
\hline
\end{tabular}

and $1.2_{-0.4}^{+0.5}$ sources in the soft and hard band, respectively, while we detect one and two sources. Moreover, in the hard $\mathrm{X}$-ray band within the sensitivity limits of Elais-S1, we expect to detect $0.2 \pm 0.1$ sources in the Lockman Hole and $0.03 \pm 0.01$ sources in the HDF-N while no sources have been detected in these two surveys.

In conclusion, the survey in the Lockman Hole is intermediate between the surveys in the Elais-S1 and HDF-N fields. Due to its large sky coverage, the Elais-S1 survey picks up very powerful and rare hard-X ray sources. On the other hand, the deep X-ray survey in the HDF-N allows the detection of very faint X-ray sources, and therefore also normal and starburst galaxies, in a small region of sky. So far, only the survey in the Lockman Hole region has sufficient depth and sky coverage to study a representative population of AGNs detected in the ISOCAM mid-IR surveys.

\section{Properties of the X-ray-mid-IR matched sources}

Optical colours, redshifts and spectral classifications are available for many of the galaxies emitting in X-ray and mid-IR bands. In the case of the Lockman Hole, the best known galaxies are those already detected with ROSAT (see Lehmann et al. 2000, 2001), which constitute approximately half of our sample. On the contrary, redshifts are 
Table 4. List of the Chandra sources with mid-IR counterparts in the Hubble Deep Field and flanking fields ordered with decreasing 0.5-2 keV flux. For each association we report: the number of Brandt et al. (2001a) Chandra source and the name of the ISOCAM counterpart (Aussel et al. 1999) (1); the J2000 coordinates $(2,3)$ of the Chandra source; the offset between associated sources in arcseconds and the probability of random association in $10^{-3}$ units for X-ray vs. optical (4, 5), mid-IR vs. optical $(6,7)$ and X-ray vs. mid-IR $(8,9)$, respectively; the $V, I$ magnitudes $(10,11)$ from Barger et al. (1999), the $R$ and $K s$ magnitudes $(12,13)$ from Hogg et al. (2000); the X-ray fluxes in the soft $(0.5-2 \mathrm{keV})$ and hard $(2-8 \mathrm{keV}) \mathrm{X}$-ray bands in $10^{-15} \mathrm{cgs}$ units $(14,15)$; the $2-10 \mathrm{keV}$ luminosity (16); the mid-IR fluxes at $6.75 \mu \mathrm{m}$ (17) and $15 \mu \mathrm{m}$ (18) in mJy (from Aussel et al. 1999, 2002); the $\alpha_{\mathrm{IX}}$ index described in the text (19); the measured redshift (20); the spectral classification (21) by Cohen (2000, 2001) and the AGN type (22). A dash in Col. (6) indicates that the source is not in the $L W 2$ field. The source marked with the label "Var" is a variable X-ray source detected by Brandt et al. (2001) and below the detection threshold in more recent observations (Brandt et al. 2001a). Sources detected in the ultra-hard band (4-8 keV) are marked with an asterisk on the hard-band flux. For the source \#172 we recompute $I$ and $V$ magnitudes on the images of Barger et al. (1999) since the original catalogue lists one source instead of two close sources. We classified the spectra of \#178, \#190 and \#Var according to Cohen (2000) by inspecting the reduced spectra of Barger et al. (1999). Redshifts sources are: (0) Hawaii group, (1) Cohen et al. (1996), (2) Lowental et al. (1997), (3) Hogg et al. (1997), (4) Waddington et al. (1999), (5) Cohen et al. (2000), (6) Dawson et al. (2001).

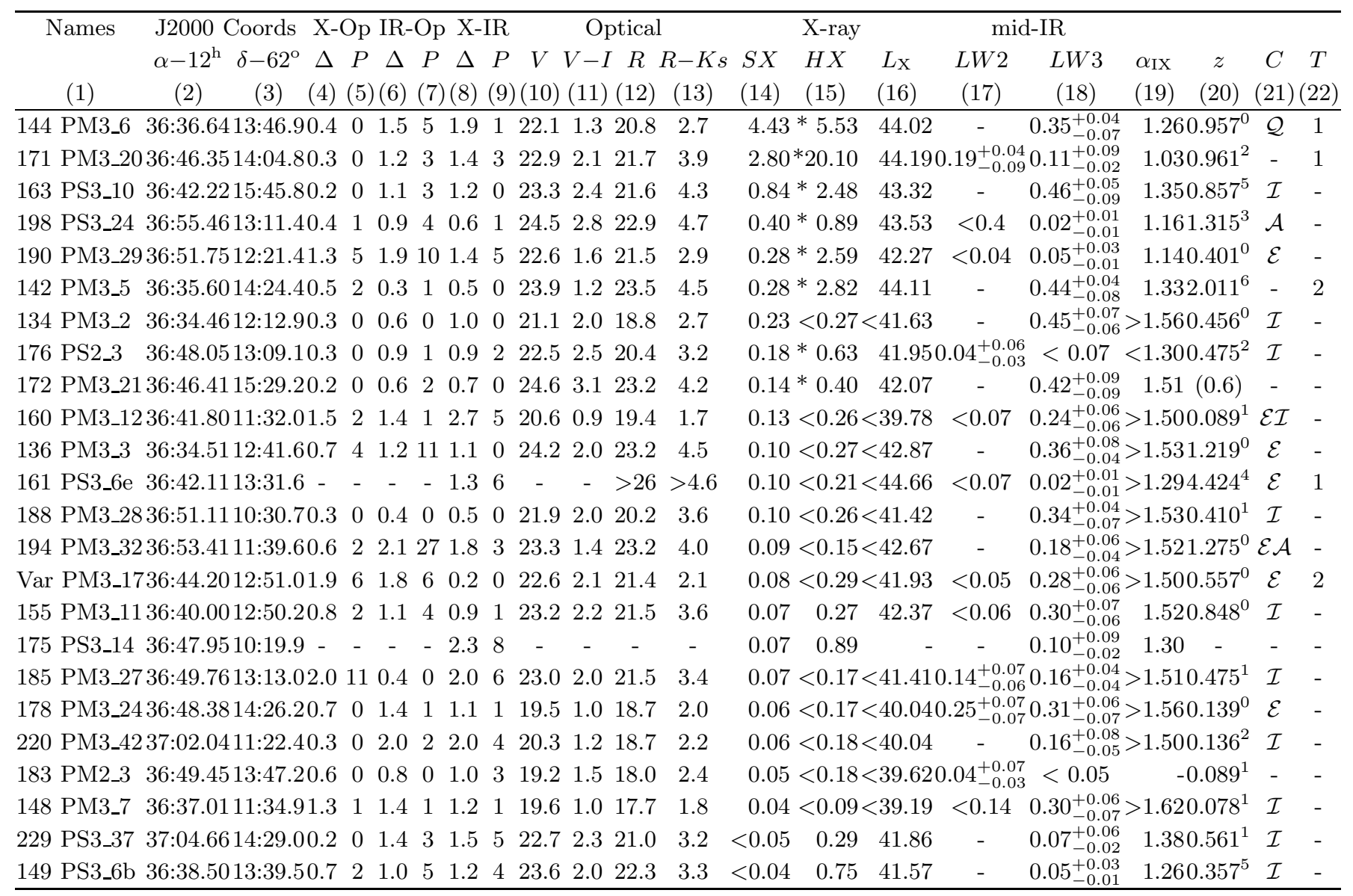

known for all but two of the galaxies of the HDF-N sample due to the great efforts made in this area (e.g. Hogg et al. 2000; Cohen et al. 2000), although only few galaxies are classified as AGNs or starburst galaxies according to their spectral features. In this Section we analyse the sources both detected in the mid-IR and X-ray from the point of view of their optical, X-ray and infrared emissions. By comparing their properties with those of local template galaxies we are able to classify these sources as starburst - or AGN-dominated.

\subsection{Optical properties}

The HDF-N survey, which is the deepest we consider here, allows one also to detect very faint sources and thus starburst and nearby galaxies (see Hornschemeier et al. 2001; Elbaz et al. 2002). Only a small part of the sources detected both in the mid-IR and X-rays are optically classified as AGN (20\%). Also the redshift distribution of these galaxies reflects this situation. The median redshift of 0.5 is typical of the mid-IR galaxy population (see Fadda et al. 2002; Flores et al. 2002), while the median redshift of 

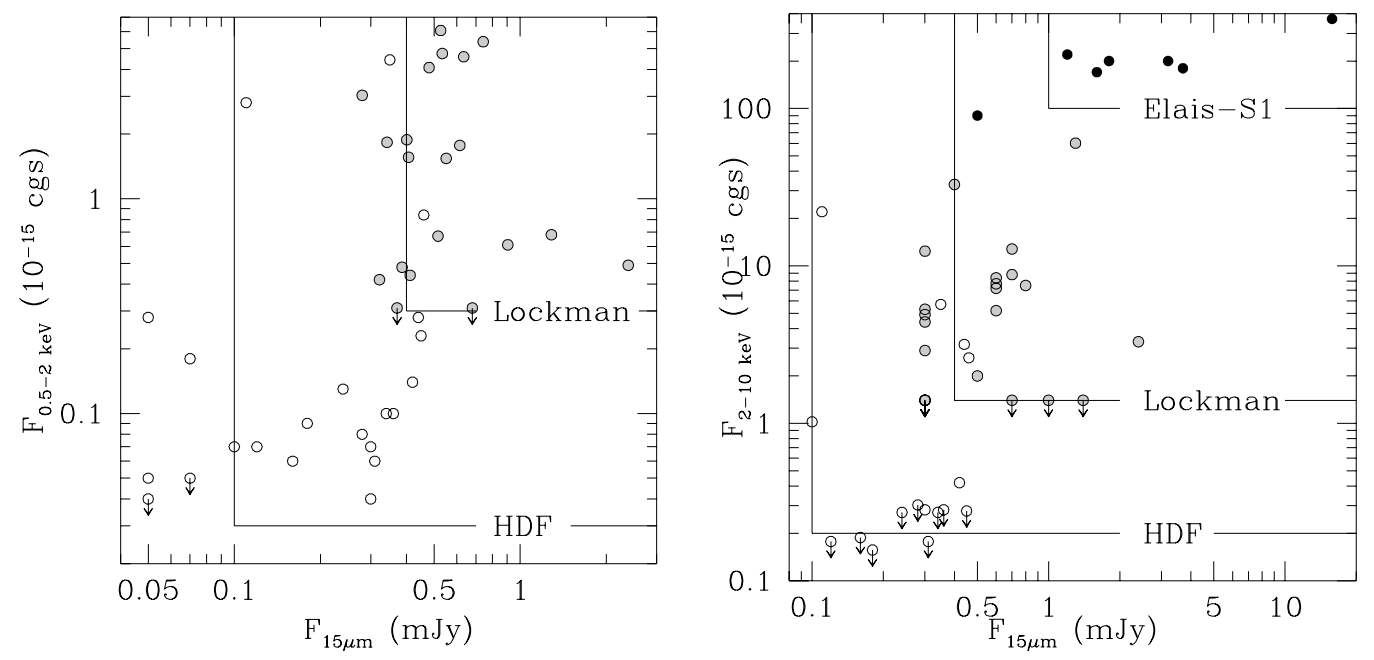

Fig. 4. Mid-IR vs. X-ray fluxes for galaxies observed in the Elais-S1 (black), Lockman (grey) and HDF-N (white) surveys. Diagrams refer to the soft band $(0.5-2 \mathrm{keV})$ on the left and to the hard band $(2-10 \mathrm{keV})$ on the right. The lines delimit the X-ray sensitivity and the mid-IR $(15 \mu \mathrm{m}) 80 \%$ completeness limits.
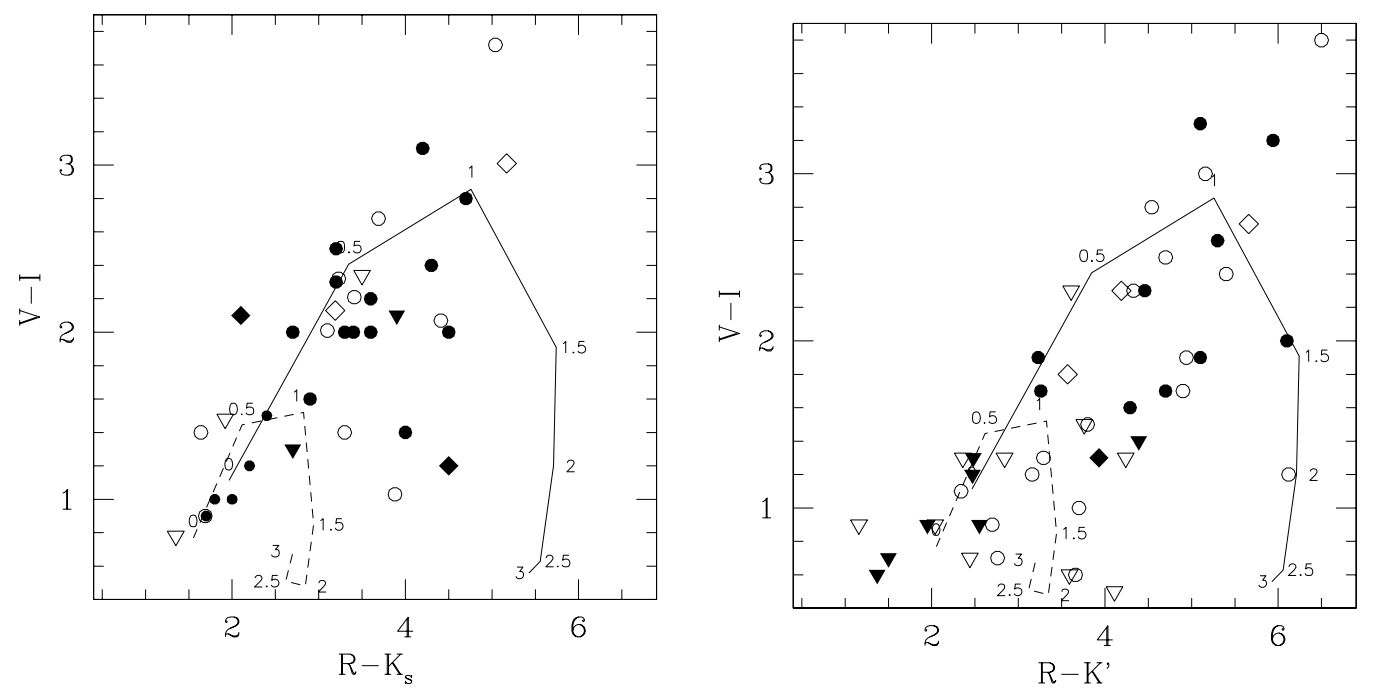

Fig. 5. Optical colour diagrams for X-ray-emitting galaxies in the part of HDF-N (left) and Lockman Hole fields (right) surveyed in X-ray and mid-IR. Triangles, diamonds and circle refer to type-1 AGNs, type-2 AGNs and unknown type galaxies, respectively. X-ray sources detected in the mid-IR are marked with full symbols. Dashed and solid lines show colours of spiral and elliptical galaxies, respectively, as a function of the redshift. The curves are computed using the PEGASE2 code (Fioc \& Rocca-Volmerange 1997). Sources with $L_{\mathrm{X}}<10^{41} \mathrm{erg} \mathrm{s}^{-1}$ in the HDF-N are indicated with smaller symbols and lie around the lines of normal galaxies.

the galaxies classified as AGN is 1 . On the contrary, the sources detected in the Elais-S1 survey are almost exclusively AGN at high redshift. Excluding a normal galaxy detected at $z=0.3$, all the other sources lie at $z>0.4$ with a median value of $z=2$.

Finally, among the sources detected in the Lockman Hole almost half of the sample is classified as AGN while the rest is up to now of unknown type. The median redshift of the sources is $z=1$ and all the sources lie at $z>0.4$. Therefore, this population of galaxies differs from the bulk of the galaxies detected in the Lockman Hole, which lie at a redshift of 0.6 (see Fadda et al. 2002). We can learn something more about the spectrally unclassi- fied galaxies by looking at the optical colour diagrams (see Fig. 5). As expected, the type-1 AGNs cluster in a region of blue colors while the type- 2 AGNs are in general redder and less clustered on the diagram. Many of the galaxies with unknown type lie in the region occupied by type-2 AGNs, suggesting that they are highly extincted objects and probably most of them are type-2 AGNs.

To aid in the interpretation of the diagrams, we overlay two galaxy tracks corresponding to elliptical and spiral templates. These models were produced with the PEGASE2.0 code ${ }^{2}$ (Fioc \& Rocca-Volmerange 1997)

\footnotetext{
${ }^{2}$ http://www.iap.fr/users/fioc/PEGASE.html
} 

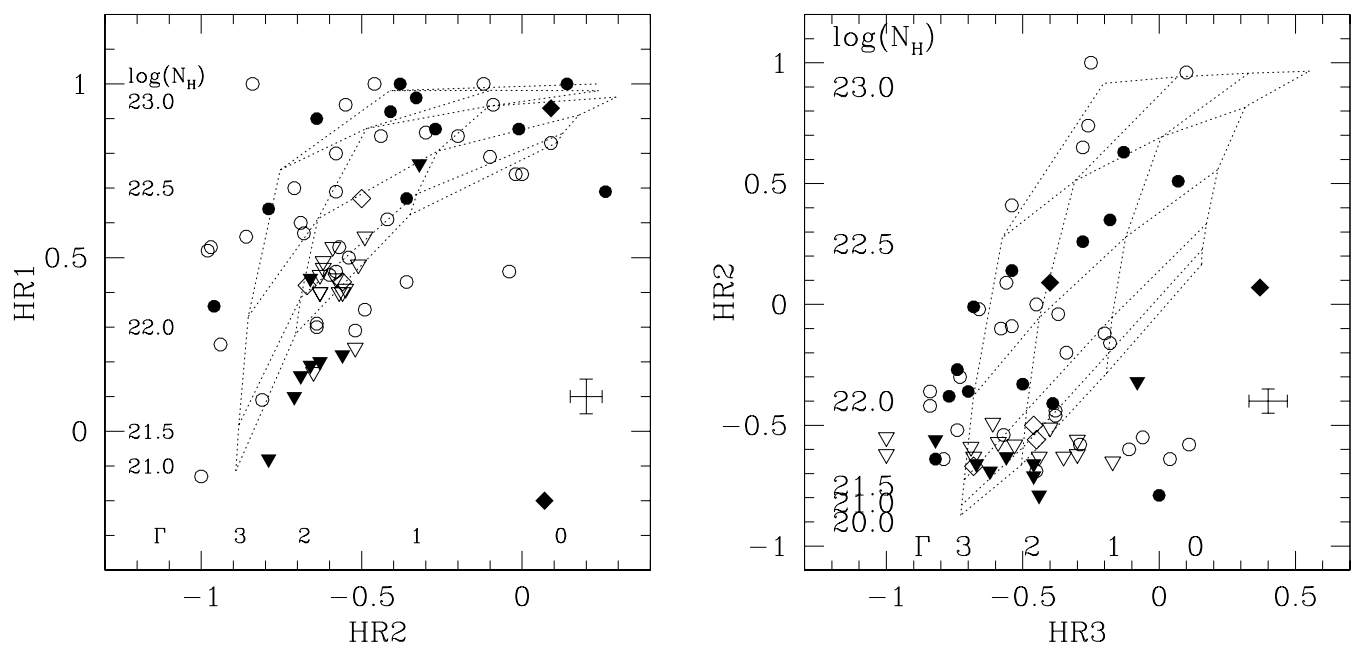

Fig. 6. X-ray diagnostic diagrams based on hardness ratios (see Hasinger et al. 2001). Triangles, diamonds and circle refer to type-1 AGNs, type-2 AGNs and unknown-type galaxies, respectively. Galaxies inside the common X-ray and mid-IR area are shown. The cross indicates the median error bar of the points. Only points with error less than 0.1 are plotted. The galaxies with mid-IR emission are marked with full symbols. For these galaxies $H R 1, H R 2$ and $H R 3$ values are reported in Cols. 16-18 of Table 3. The grid gives the expected hardness ratios for power-law models with different values of the photon index $\Gamma$ and of the neutral hydrogen absorption $\log N_{\mathrm{H}}$ (in the observed frame).

assuming a Salpeter initial mass function with standard cutoff $\left(0.1-120 M_{\odot}\right)$. For the elliptical track we adopt a star formation timescale of $1 \mathrm{Gyr}$, observed at $6 \mathrm{Gyr}$, without extinction and nebular emission. For the spiral track we consider a star formation timescale of $5 \mathrm{Gyr}$, observed at $3 \mathrm{Gyr}$, extinction with disk geometry and no nebular emission. Tracks are labelled with representative redshifts over the range $0<z<3$, which corresponds to the redshift range of the galaxies observed. Few galaxies appeared clustered around these lines. In particular, five galaxies detected in the HDF-N with low X-ray luminosities have colours typical of normal galaxies. Most of the galaxies are scattered over the diagram, but there are almost no galaxies which follow the track of the elliptical galaxies with $z>1$.

Finally, we note that our sample of XMM-ISO matched sources contains five highly obscured sources that are extremely red objects (i.e. EROs, according to the definition $R-K \geq 5$ ). Objects of this type are claimed to constitute about $30 \%$ of the optically faint X-ray sources in the deep Chandra survey of the HDF-N (Alexander et al. 2001a). On the other hand, Pierre et al. (2001) showed that is possible to select this kind of objects using midIR observations. This sample of objects will be studied in more detail by Franceschini et al. (2001). In the Lockman field, another four EROs were detected by XMM-Newton and not by ISOCAM. As discussed in Franceschini et al. (2001), the expected $15 \mu \mathrm{m}$ fluxes of these objects fall below the detection limit of the survey ( $0.3 \mathrm{mJy})$.

\subsection{X-ray diagnostic}

Thanks to the large energy range which can be explored with XMM-Newton it is possible to construct colour- colour X-ray diagrams and to classify sources on the basis of their X-ray spectra alone (Hasinger et al. 2001). Figure 6 shows X-ray spectral diagnostic diagrams based on the hardness ratios computed using four independent energy bands. The hardness ratios are obtained with the formula $H R=(H-S) /(H+S)$, where $H$ and $S$ correspond to the counts in the harder and softer energy bands, respectively. $H R 1, H R 2$ and $H R 3$ compare the $0.2-$ 0.5 vs. $0.5-2 \mathrm{keV}, 0.5-2$ vs. $2-4.5 \mathrm{keV}$, and $2-4.5$ vs. $4.5-$ $10 \mathrm{keV}$ bands, respectively. A grid representing the expected hardness ratios for power-law models with different values of photon index $(\Gamma)$ and hydrogen absorption $\left(\log N_{\mathrm{H}}\right)$ computed in the observed frame is superimposed on the data. The populations of type- 1 and type- 2 AGNs occupy different regions in these diagrams. In particular, AGN-1 galaxies populate a limited portion of the diagrams in the soft range (and a particularly narrow $H R 2$ range) while the new XMM-Newton galaxies and known AGN-2 type galaxies have harder spectra than those of AGN-1 galaxies and occupy a larger area (see discussion in Hasinger et al. 2001). Also in this case, most of new XMM-Newton galaxies detected in the mid-IR lie in a clearly separated region with respect to the type-1 AGNs.

If we admit that unclassified galaxies are all type2 AGNs, we detect at $15 \mu \mathrm{m}$ at the $3 \sigma$ level 7 AGN-1 galaxies and 15 AGN-2 galaxies (only three of these are classified as AGN-2). Although the statistics are poor, the fraction of AGN source types matches that found in the CFRS field 1415+52 using a multi-wavelength method to classify the galaxies. In this case, studying a sample of 19 ISOCAM sources, Flores et al. (1999) classified two sources as AGN-1 and three as AGN-2. For two other sources the classification as AGN-2 or starburst galaxies is equally probable. 

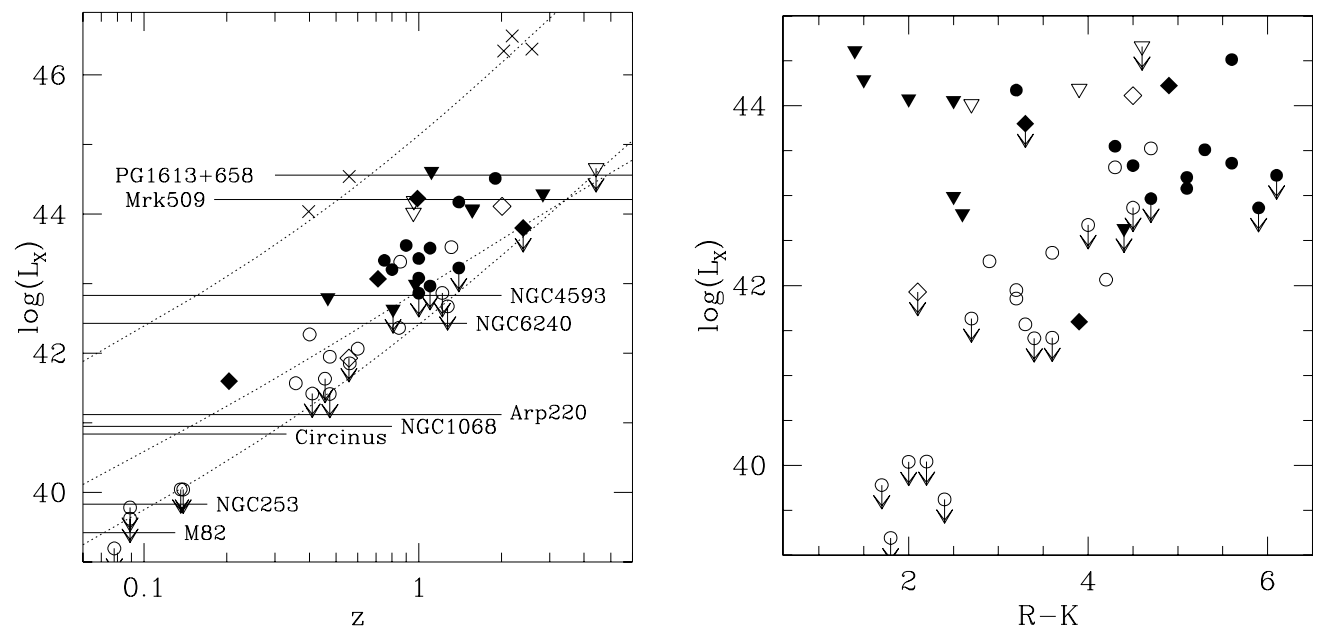

Fig. 7. 2-10 keV rest-frame luminosity versus redshift (left) and $R-K$ colour (right) for the X-ray mid-IR matched sources. Open symbols, grey symbols and crosses refer to the HDF-N, Lockman and Elais surveys, respectively. Type-1 and type-2 AGNs are marked with triangles and squares, respectively, while circles identify unclassified sources. In the left figure, the three dashed lines show the sensitivity limits of the three X-ray surveys. The horizontal lines trace the X-ray luminosity of the template galaxies discussed in the text. The luminosity distance is computed according to Carroll et al. (1992) assuming a cosmology of $H_{0}=70 \mathrm{~km} \mathrm{~s}^{-1} \mathrm{Mpc}^{-1}, \Omega_{\lambda}=0.7$ and $\Omega_{\mathrm{M}}=0.3$.

\subsection{Comparison with local templates}

Detailed spectral energy distributions (SEDs) have been obtained in the hard X-ray band (with ASCA and BeppoSAX) and in mid- to far-IR (with ISO) for few local galaxies which are representative of the classes of objects found in our samples.

Before analysing X-ray luminosities and X-ray to midIR spectral indices of the galaxies of our samples, we discuss the template galaxies which will be compared with our data.

Type-1 AGNs. Mrk 509 and NGC 4593 have been chosen as typical Seyfert 1 galaxies (data from Clavel et al. 2000; Perola et al. 2000; Guainazzi et al. 1999), while PG $1613+658$ has been taken as representative of radio-quiet quasars (data from Haas et al. 2000; Lawson \& Turner 1997).

Type-2 AGNs. This class of objects is expected to be easily detected by combined hard X-ray and mid-IR surveys, since almost all the UV and soft X-ray emission of the nucleus is reprocessed into infrared light. We consider four examples with different column densities. NGC 1068, the archetypal object for the class of Seyfert 2 galaxies (data from Sturm et al. 2000; Matt et al. 1997), has an extreme Compton-thick nucleus $\left(N_{\mathrm{H}}>10^{25} \mathrm{~cm}^{-2}\right.$; Matt et al. 1997). Due to this fact, it has a mid-IR to X-ray flux spectral index which is more typical of starburst galaxies than type-2 AGNs (see Fig. 8). We consider two moderately Compton-thick Seyfert 2s: Circinus and NGC 6240 $\left(N_{\mathrm{H}} \sim 4.3 \times 10^{24} \mathrm{~cm}^{-2}\right.$, Matt et al. 1999a, and $N_{\mathrm{H}} \sim$ $2 \times 10^{24} \mathrm{~cm}^{-2}$, Vignati et al. 1999, respectively). Circinus is a Seyfert 2 object with a reflection-dominated spectrum in the $2-10 \mathrm{keV}$ range and a transmitted component above $10 \mathrm{keV}$ (data from Sturm et al. 2000; Siebenmorgen et al. 1997; Matt et al. 1999; Sambruna et al. 2001). NGC 6240 (data from Charmandaris et al. 1999;
Vignati et al. 1999), according to Vignati et al. (1999) is dominated by the AGN and not from star formation, as deduced by Genzel et al. (1998) on the basis of the ISO spectrum. Finally, we show the Compton-thin luminous IRAS source IR 23060+0505 (data from Brandt et al. 1997 and from the ISO archive) which has $N_{\mathrm{H}} \sim 10^{22} \mathrm{~cm}^{-2}$ (Brandt et al. 1997).

Starbursts. M 82 and NGC 253, two of the nearest starburst galaxies, are assumed as typical templates for galaxies with active star formation (data from Sturm et al. 2000; Cappi et al. 1999).

Ultraluminous galaxies. Galaxies of this class, which emit large parts of their bolometric luminosity in the infrared, are known to be powered mainly by star formation, although a small fraction of the emission is probably due to AGN activity (e.g. Lutz et al. 1998; Tran et al. 2001). We chose Arp 220 as an example of an ultraluminous starburst galaxy (data from Sturm et al. 1996; Charmandaris et al. 1999; Iwasawa et al. 2001).

\subsection{X-ray luminosities}

Since we have spectroscopic and photometric redshifts for almost all the galaxies of our samples, it is possible to compute X-ray luminosities of these galaxies and compare them with those of local templates.

To compute the luminosity distance we assume a cosmology of $H_{0}=70 \mathrm{~km} \mathrm{~s}^{-1} \mathrm{Mpc}^{-1}, \Omega_{\lambda}=0.7$, and $\Omega_{\mathrm{M}}=0.3$ using the formula in Carroll et al. (1992). As we can note in Fig. 7, the sources in the Lockman Hole sample have $2-10 \mathrm{keV}$ rest-frame luminosities between $10^{42.5} \mathrm{erg} \mathrm{s}^{-1}$ and $10^{45} \mathrm{erg} \mathrm{s}^{-1}$, which are typical of luminous type-2 AGNs and normal type-1 AGNs. Sources detected in the Elais-S1 have luminosities typical of type-1 AGNs. Finally, among the sources detected 

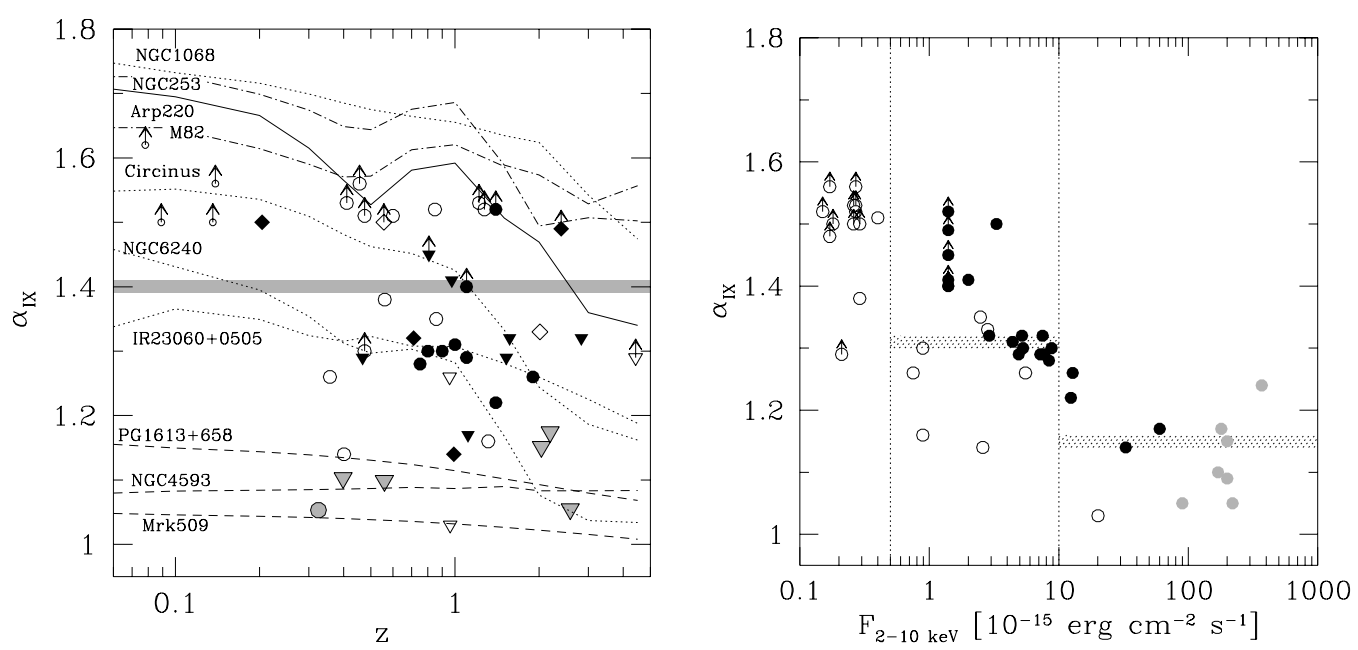

Fig. 8. On the left: distribution of the observed mid-IR to hard X-ray spectral indices $\alpha_{\mathrm{IX}}$ as a function of redshift. Open, grey and black symbols refer to objects detected in the HDF-N, Elais-S1 and Lockman Hole surveys, respectively. Triangles, diamonds and circles represent type-1 AGNs, type-2 AGNs and unknown types, respectively. HDF-N galaxies with $L_{\mathrm{X}} \leq 10^{40}$ erg s ${ }^{-1}$ are drawn with small symbols. Templates derived from various types of known active and starburst galaxies are shown (see the text). The horizontal band shows the $\alpha_{\mathrm{IX}}$ of the cosmic background. On the right: $\alpha_{\mathrm{Ix}}$ versus the $2-10 \mathrm{keV}$ flux. Median values in the flux ranges assumed to compute the AGN contribution to the mid-IR background are drawn.

by Chandra in HDF-N, we find low luminosities sources at low redshift which are probably starburst galaxies, few galaxies in the luminosity range populated by Lockman sources, and a population of galaxies with intermediate luminosities which could be ultraluminous infrared galaxies or low-luminosity type-2 AGNs.

In the same Fig. 7 we plot also the hard X-ray luminosity versus the $R-K$ colour. This allows one clearly to segregate normal galaxies which are faint X-ray sources and $R-K \sim 2$ and type-1 AGNs, which populate the left upper corner of the diagram. It is still difficult to distinguish type-2 AGNs from ultraluminous galaxies.

\subsection{Mid-IR to X-ray spectral index}

A way to combine the information coming from mid-IR and X-ray fluxes is to compute the mid-IR to X-ray spectral index $\alpha_{\text {IX }}$ assuming a power law spectral energy distribution: $F_{\nu} \propto \nu^{-\alpha_{\mathrm{IX}}}$. Values reported in Tables 3 and 4 are computed using the observer frame flux densities at $15 \mu \mathrm{m}$ and $5 \mathrm{keV}$. The flux densities at $5 \mathrm{keV}$ have been derived from the observed $2-10 \mathrm{keV}$ fluxes $(2-8 \mathrm{keV}$ in the case of HDF-N Chandra data) and spectral indices.

In Fig. 8, which gives $\alpha_{\mathrm{IX}}$ as a function of redshift, we report all the sources detected in the Lockman, HDF$\mathrm{N}$ and Elais-S1 surveys. We also show the values of $\alpha_{\mathrm{IX}}$ as a function of redshift for the aforementioned local templates.

Galaxies dominated by star formation (starburst and ultraluminous galaxies) have high values of $\alpha_{\text {IX }}$ at any redshift. On the contrary, type-1 AGNs have quasi-constant values between 1 and 1.2. Between these two envelopes of curves we find the templates of moderately Compton-thick and Compton-thin type-2 AGNs. Only the type-2 Seyfert NGC 1068, which has an extreme Compton-thick nucleus, lies in a region of the diagram occupied by starburstdominated galaxies. In fact, if the column density exceeds $10^{25} \mathrm{~cm}^{-2}$ the nuclear radiation is heavily obscured also in the hard X-ray band (see e.g. Matt et al. 2000).

Most of the galaxies detected in the Lockman Hole survey populate the region of the diagram delimited by moderately Compton-thick and Compton-thin type-2 AGNs. The galaxies detected in the Elais-S1 surveys lie around the type-1 AGN curves.

The HDF-N survey, due to its high sensitivity, is able to detect also non-active galaxies with high $\alpha_{\text {IX }}$ index. In fact, half of the HDF-N sources lie just below the curves of starburst and ultraluminous galaxies, while the other half have $\alpha<1.4$. Combined with the information on the $\mathrm{X}$-ray luminosity, we will use this diagram to discriminate between HDF-N sources whose emission is dominated by AGN or star formation activity.

It is interesting to remark that a large part of the type1 AGNs detected in the Lockman area have an $\alpha_{\text {IX }}$ index greater than those of the local templates. Except for one case which is an absorbed type-1 AGN, as revealed by the X-ray hardness ratio diagrams (\#79 in Table 3), the most probable explanation is that star formation of the host galaxies contribute a large fraction of the midIR flux. Hence, their $\alpha_{\text {IX }}$ values should differ significantly from those of local templates, for which we can easily discriminate between the host galaxy and AGN.

\section{Contribution to the extragalactic background light}

The samples of sources discussed allow us to estimate how much of the mid-IR extragalactic light detected in the mid-IR surveys is due to AGNs. We can derive this quantity in a direct way by simply computing the total of the mid-IR fluxes of the sources whose emission 
is dominated by AGNs and dividing this by the total of the mid-IR fluxes of the sources in the area. In this case, we can estimate only the AGN contribution within the sensitivity limits of the surveys and not to the total extragalactic background mid-IR light (more than 30\% and $60 \%$ of the $15 \mu \mathrm{m}$ background is resolved at the flux limits of the Lockman Hole and HDF-N surveys, respectively). Alternatively, we can use the median $\alpha_{\text {IX }}$ for different classes of contributors to the X-ray background to estimate the total contribution to the mid-IR extragalactic background (following Severgnini et al. 2000).

\subsection{Estimating the AGN contribution to mid-IR surveys}

We can derive the AGN contribution in the case of HDF$\mathrm{N}$ and Lockman surveys for which we have the complete information on X-ray and mid-IR sources. To do this, we have to select on the basis of the optical, X-ray and midIR properties, the subsamples of sources whose mid-IR emission is dominated by AGNs.

In the case of the Lockman Hole, we have seen that almost all the galaxies in the sample have high X-ray luminosity and low $\alpha_{\text {IX }}$ values. Therefore, we conservatively assume that the mid-IR emission of all the galaxies in the sample is due to AGNs.

In the case of the HDF-N, we have seen that Chandra observations are so deep that X-ray emission from starburst galaxies is also detected. Thus, in order to estimate the AGN contribution to the mid-IR total emission we have to select the galaxies whose mid-IR emission is dominated by the AGN. We base our selection on the X-ray luminosity (see Fig. 7) and on the shape of the SED from radio to X-ray wavelengths (see Fig. 9).

Out of 16 sources with flux greater than $0.1 \mathrm{mJy}$, four have a high X-ray luminosity $\left(L_{\mathrm{X}}>10^{43} \mathrm{erg} \mathrm{s}^{-1}\right)$ and another four are faint in the X-ray $\left(L_{\mathrm{X}} \leq 10^{40} \mathrm{erg} \mathrm{s}^{-1}\right)$. In these cases, we assume that the mid-IR emission is dominated by AGN and star-formation activity, respectively. Moreover, since the source \#172 is detected also in the ultra-hard band, we consider that it is dominated by AGN activity. We classify all the other sources, which have an intermediate X-ray luminosity, by comparing their radio, mid-IR, near-IR, optical and X-ray data with the SEDs of two star formation-dominated and two type-2 AGNs (Arp 220, M 82, Circinus and NGC 6240) for which we have the SED from radio to X-ray frequences. Radio data at 8.5 GHz come from Richards et al. (1998) and at 1.4 GHz from Richards (2000). In the $1.4 \mathrm{GHz}$ case, we also retrieved the image ${ }^{3}$ to estimate the $1.4 \mathrm{GHz}$ flux of the source \#155. For each galaxy we fitted the data to the template SEDs scaled in luminosity choosing the fit with the lowest $\chi^{2}$ value. Figure 9 shows the $\chi^{2}$ values for each SED and the the best fit superimposed to the data. In the fit we considered also upper limits on the hard X-ray fluxes. This means that the $\chi^{2}$ of the NGC 6240

\footnotetext{
${ }^{3}$ http://www.cv.nrao.edu/ jkempner/vla-hdf/
}

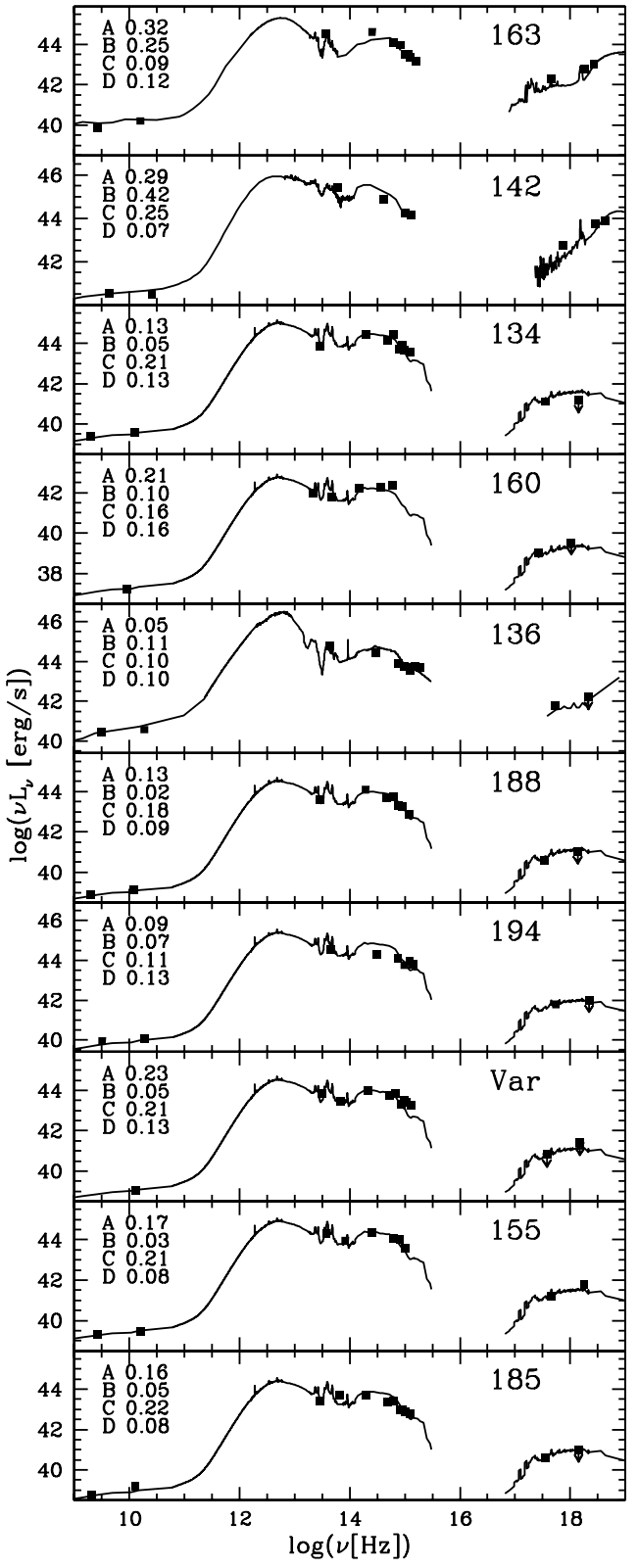

Fig. 9. Radio, mid-IR, near-IR, optical and X-ray data for sources in the HDF-N (numbered as in Table 4) superimposed to scaled SEDs of template galaxies (A: Arp 220, B: M 82, C: NGC 6240, D: Circinus). The fit with the lowest $\chi^{2}$ (see values in the upper left corner) is shown.

and Circinus SEDs are typically underestimated. For comparison, in the same figure, the SED of two bright $\mathrm{X}$ ray sources (\#163 and \#142) and one faint X-ray source (\#160) are also shown (the best fit is obtained with the NGC 6240, Circinus and M 82 SEDs, respectively). The sources with intermediate X-ray luminosity are all well fitted with the M82 SED, except for \#136 which is fitted by the Arp 220 SED. We note that also the variable X-ray source ("Var") follows the M 82 SED very well. Therefore, in these cases we assume that the mid-IR emission of these sources is not dominated by the presence of an AGN. 

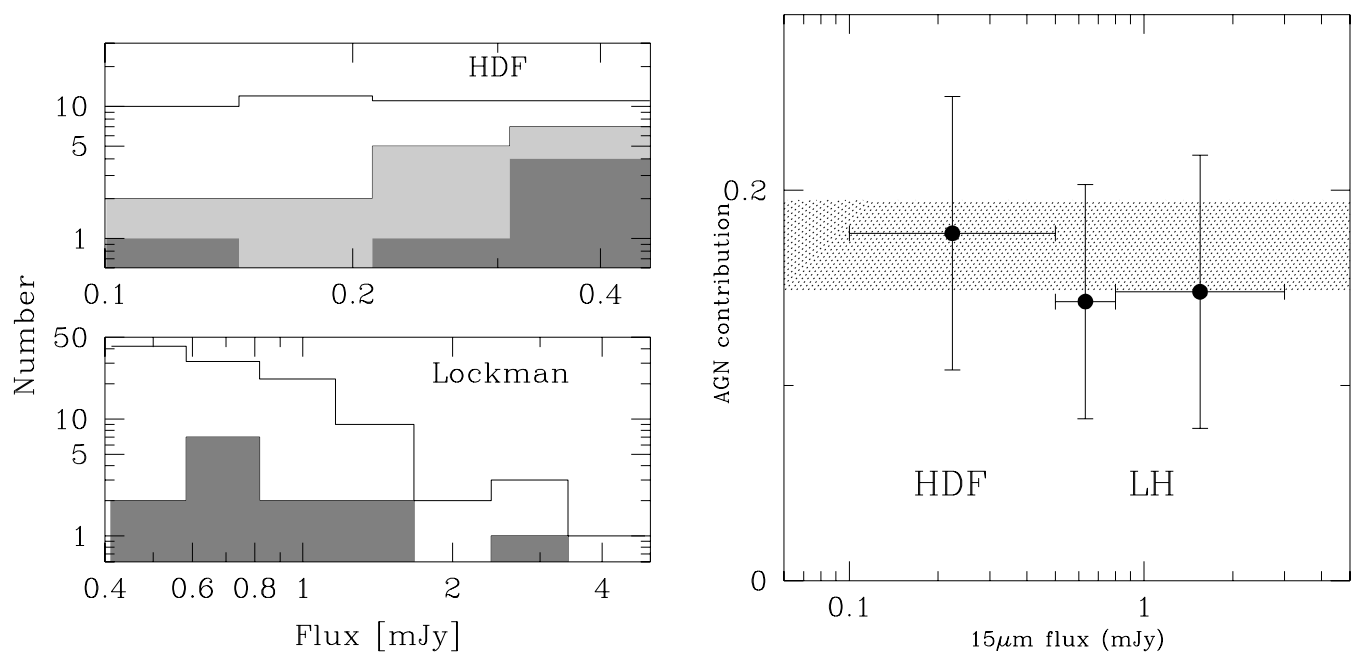

Fig. 10. On the left: histogram of $L W 3$ fluxes in the Lockman and HDF-N surveys. Sources detected in the X-ray are shaded, while the black histogram shows the sources dominated by AGN emission. On the right: ratio of integrated $15 \mu \mathrm{m}$ flux of AGN-dominated sources to that of all the mid-IR extragalactic sources as a function of limiting flux. The $0.1 \mathrm{mJy}$ points comes from the HDF-N survey, while the other points are computed on the basis of the Lockman survey. The horizontal band shows the percentage of background light due to AGN emission according to the analysis based on the $\alpha_{\mathrm{IX}}$ index (see the text).

In Fig. 10 we summarise the contribution of AGNs to the mid-IR extragalactic background as a function of the flux. The bin between 0.1 mJy and 0.5 mJy has been defined using the HDF-N data, since the sources detected in this field cover this range of fluxes well and the HDF-N is complete for fluxes greater than $0.1 \mathrm{mJy}$ (Aussel et al. 1999). Due to its small size, there are no sources in the HDF-N with fluxes greater than $0.5 \mathrm{mJy}$. Hence, the contribution in the other two bins is based on the Lockman Hole data which are more than $80 \%$ complete at the flux of $0.5 \mathrm{mJy}$ (Fadda et al. 2002).

In the HDF-N there are 42 sources for a total of $9.9 \mathrm{mJy}$ in the $0.1-0.5 \mathrm{mJy}$ bin, five of which are classified by us as AGN-dominated (\#142, \#144, \#163, \#171 and \#172). This implies an AGN contribution in this flux bin of $(17.8 \pm 7) \%$.

The Lockman Hole survey covers well the $0.5-3 \mathrm{mJy}$ flux interval where we find 103 sources for a total of 81.8 mJy with 13 sources which are AGN-dominated, leading to a total contribution of $(14.6 \pm 4.7) \%$. In Fig. 10, we report the contribution in two bins: $0.5-0.8 \mathrm{mJy}$ $((14.3 \pm 6) \%)$ and $0.8-3 \mathrm{mJy}((14.8 \pm 7) \%)$. The contribution in this interval is probably slightly underestimated because, as is clear from Fig. 7, XMM-Newton observations may miss a population of fainter X-ray sources that contain highly obscured AGNs. The effect should not be dramatic because, as we have seen in our analysis of the HDF-N sources, the mid-IR emission of most of these intermediate $\mathrm{X}$-ray luminous sources is not dominated by the AGN activity.

From these estimates, we can derive the AGN contribution to the fraction of the mid-IR extragalactic background due to the emission of $0.1-3 \mathrm{mJy}$ sources, which constitute $\sim 70 \%$ of the measured background. The $0.1-$ $0.5 \mathrm{mJy}$ and $0.5-3 \mathrm{mJy}$ sources contribute $48 \%$ and $23 \%$ of the observed mid-IR background, respectively (Elbaz et al. 2002). Therefore, AGNs contribute $(16.8 \pm 6.2) \%$ of the fraction of the mid-IR extragalactic background for which are responsible the sources detected in the $0.1-3$ mJy flux interval.

\subsection{Estimating AGN contribution with median mid-IR to $X$-ray spectral indices}

In order to exploit all the existing information from the deep HDF-N data to the shallow ELAIS-S1 observations, we can estimate the AGN contribution to the mid-IR background using the mean $\alpha_{\mathrm{IX}}$ indices of bright and faint X-ray sources of the X-ray background with mid-IR emission (see Severgnini et al. 2000 for an application of this technique to the SCUBA sources).

To apply this method we have to know the values of the $\mathrm{X}$-ray and mid-IR backgrounds. We compute the $5 \mathrm{keV}$ X-ray background using the estimation of the 1-7 keV background by Chen et al. (1997), which is in good agreement with recent Chandra and XMM counts. In particular, the counts by Brandt et al. (2001a) clearly flatten at low fluxes, indicating that almost all the background is resolved in this survey. Assuming the background of Chen et al. (1997), Alexander et al. (2001a) evaluate that $\sim 86 \%$ of the $2-8 \mathrm{keV}$ background is resolved by Chandra observations in the HDF-N region. The recent estimation by Vecchi et al. (1999) with Beppo-SAX observations seems to be too high to agree with recent deep observations of XMM and Chandra satellites. In the case of $15 \mu \mathrm{m}$, the total background has not yet been measured. Observational values are the upper limit of $5 \mathrm{nW} \mathrm{m}{ }^{-2} \mathrm{sr}^{-1}$ established by Stanev \& Franceschini (1998) measuring the optical depth at high energies due to the $\gamma \longrightarrow \gamma$ interaction with the background infrared photons and 
the lower limit of $\left.\nu I(\nu)\right|_{15 \mu \mathrm{m}}=2.4 \mathrm{nW} \mathrm{m}{ }^{-2} \mathrm{sr}^{-1}$ obtained by Elbaz et al. (2002) integrating the flux of all the sources in the deep ISOCAM surveys down to the flux limit of 0.05 mJy. Franceschini et al. (2001), on the basis of their evolutionary model, which takes into account counts in the mid-IR, far-IR and sub-mm, and measurement of the far-IR background, expect that the contribution of fainter sources would bring the total background to $\left.\nu I(\nu)\right|_{15 \mu \mathrm{m}}=3.3 \mathrm{nW} \mathrm{m}{ }^{-2} \mathrm{sr}^{-1}$. This value, which is not far from values predicted by other models (Chary \& Elbaz 2001; Xu 2000) and from values found by Altieri et al. (1999) using cluster-lensed data, has been adopted in our analysis.

The horizontal band in Fig. 8 represents the $\alpha_{\text {IX }}$ of the cosmic background, assuming that most of the flux in the two spectral windows comes from sources with a similar distribution of redshifts centred around $z=1$. Therefore, this value should correspond to the mid-IR to X-ray index of the population which dominates the X-ray background if the same population were responsible for the totality of the mid-IR background. Otherwise, fitting both backgrounds requires a combination of AGN and star formation activity.

The flattening of the $2-8 \mathrm{keV}$ counts in the HDF$\mathrm{N}$ deep Chandra survey (Brandt et al. 2001a) clearly shows that almost all the hard X-ray background is resolved at the sensitivity of this survey. Since these counts agree very well with the counts by Mushotzky et al. (2000), extrapolating their result we can say that about $85 \%$ of the $2-10 \mathrm{keV}$ background is resolved at a flux of $0.5 \times 10^{-15} \mathrm{erg} \mathrm{s}^{-1} \mathrm{~cm}^{-2}$ (see also Alexander et al. 2001a). We do not consider sources with fluxes less than this value because most of them have only upper limits on the flux and are probably starburst galaxies (according to their low X-ray luminosity).

To evaluate the AGN contribution to the mid-IR background we divide the sources in two groups according to their X-ray fluxes: sources brighter than $10^{-14} \mathrm{erg} \mathrm{s}^{-1} \mathrm{~cm}^{-2}$ and faint sources with $2-10 \mathrm{keV}$ flux in the range $0.5 \times 10^{-15}-10^{-14} \mathrm{erg} \mathrm{s}^{-1} \mathrm{~cm}^{2}$. In these flux ranges the sources have similar $\alpha_{\mathrm{IX}}$ values (see Fig. 8). Using the counts of Brandt et al. (2001a) and the results of Ueda et al. (1999) and Mushotzky et al. (2000), sources brighter than $10^{-14} \mathrm{erg} \mathrm{s}^{-1} \mathrm{~cm}^{-2}$ contribute $40 \pm 10 \%$ of the hard X-ray background, while sources with flux in the range $0.5 \times 10^{-15}-10^{-14} \mathrm{erg} \mathrm{s}^{-1} \mathrm{~cm}^{2}$ contribute $45 \pm 5 \%$ of the hard X-ray background.

We can evaluate the AGN contribution to the mid-IR background by means of the median spectral indices of bright and faint X-ray sources. Bright sources, most of them are in the Elais-S1 survey, have a median $\alpha_{\mathrm{IX}}$ of 1.15 , which corresponds to only $6 \%$ of the value required to fill the mid-IR background. Therefore, bright hard Xray sources contribute to the mid-IR background ( $40 \pm$ $10) \% \times 6 \%=(2.4 \pm 0.6) \%$, i.e. in a negligible way.

The median value of $\alpha_{\mathrm{IX}}$ for faint sources is 1.30, which corresponds to $33 \%$ of the mid-IR background. Hence, faint hard X-ray sources contribute to the mid-IR background $(45 \pm 5) \% \times 33 \%=(14.8 \pm 1.7) \%$. Combining these results, we conclude that sources making up $\sim 85 \%$ of the $2-10 \mathrm{keV}$ background contribute $(17.2 \pm 2.3) \%$ of the mid-IR background.

Considering that the infrared spectra of typical AGNs, due to a dusty-torus reprocessed emission, peak around $20 \mu \mathrm{m}$ (e.g. Granato et al. 1997), the $L W 3$ ISOCAM band is expected to be quite efficient in selecting AGNs at moderate redshifts, more than far-IR or sub-millimeter observations. Due to the more diffuse and lower-intensity energy sources, starburst spectra should peak at significantly longer infrared wavelengths, as observed. This indicates that our estimated limit of $\sim 17 \%$ of mid-IR background as due to X-ray loud AGNs may be considered as an upper limit for the AGN contribution to the CIRB energy density. This obviously cannot account for possible contribution of AGNs completely opaque below $10 \mathrm{keV}$ and longwards of $20 \mu \mathrm{m}$, i.e. hidden by extremely high column density material.

\section{Summary and conclusions}

We have presented the cross-correlation between mid-IR and X-ray observations in the Lockman Hole- and HDF$\mathrm{N}$-centred regions. ISOCAM and XMM-Newton observed a common region of more than 200 square arcminutes in the Lockman Hole. A total of 24 galaxies out of 76 XMMNewton sources in this field show mid-IR emission. In particular, the percentage of hard X-ray sources with $15 \mu \mathrm{m}$ emission is around $60 \%$. On the other hand, only around $10 \%$ of the mid-IR sources show X-ray emission in the different XMM-Newton bands. Deep Chandra observations (Brandt et al. 2001a) completely cover the ISOCAM observations of the HDF-N and flanking fields. In a region of 24 square arcminutes, $25 \%$ of the mid-IR sources have been detected in the X-ray for a total of 24 sources. A comparison of the Lockman Hole, HDF-N and Elais-S1 surveys (Alexander et al. 2001) shows that these surveys are compatible in terms of source density taking into account their respective detection limits. While the HDF$\mathrm{N}$ survey is so sensitive to the detection of even normal galaxies and the Elais-S1 survey detects only very powerful and rare type-1 AGNs, the Lockman Hole survey is able to detect a population of galaxies whose emission is mostly dominated by AGNs of types 1 and 2. In particular, thanks to the increased sensitivity of XMM-Newton with respect to ROSAT, nearly half of the sources with mid-IR emission are new XMM-Newton sources. Most of the sources which are optically studied are type- 1 AGNs. Relying on optical colours and X-ray hardness ratio diagrams, we conclude that about $70 \%$ of the detected sources are type-2 AGNs. Nevertheless, XMM-Newton observations are not deep enough to detect all the obscured AGNs in the sample as are the Chandra observations in the HDFN. Only forthcoming observations of the Lockman Hole with XMM-Newton will be able to detect the population of faint obscured X-ray sources visible with Chandra in the HDF-N. We have studied how the mid-IR to hard 
X-ray index $\left(\alpha_{\text {IX }}\right)$ varies with redshift, comparing it with the expected behaviour from local templates. Most of the Lockman sources detected lie in a region of the diagram occupied by type- 2 AGN local templates and several are optically classified as type-1 AGN. Since in general these sources do not appear highly extincted using X-ray hardness diagnostic diagrams, a possible explanation is that emission from the host galaxies contributes a fraction of their IR-optical emission greater than that of local templates.

Finally, we have evaluated how much the integrated emission of AGN contributes to the total extragalactic mid-IR background light using two independent methods. A direct estimation gives a percentage of $(15 \pm 5) \%$ for the Lockman survey $\left(0.5<F_{15 \mu \mathrm{m}}<3 \mathrm{mJy}\right)$ and a value of $(18 \pm 7) \%$ for the HDF-N survey $\left(0.1<F_{15 \mu \mathrm{m}}<0.5 \mathrm{mJy}\right)$, hence a contribution of $(17 \pm 6) \%$ in the interval of fluxes $0.1<F_{15 \mu \mathrm{m}}<3 \mathrm{mJy}$. Considering median mid-IR to $\mathrm{X}$-ray spectral indices for two hard-X flux ranges, we estimate that the population of AGNs making up $\sim 85 \%$ of the $2-10 \mathrm{keV}$ X-ray background contribute $(17 \pm 2) \%$ of the mid-IR extragalactic background.

This fraction could be higher if there exists a population of AGNs that is highly obscured at X-ray wavelengths. In particular, since the X-ray background peaks at $30-40 \mathrm{keV}$ while we can now observe only up to 5$10 \mathrm{keV}$, we expect that deeper X-ray observations (over a wider spectral range) will unveil more highly extincted AGNs. Considering that IR spectra of typical AGNs peak around $20 \mu \mathrm{m}$ while starburst spectra peak at significantly longer infrared wavelengths, this figure may be considered as an upper limit to the AGN contribution to the cosmic IR background (CIRB) energy density.

We conclude that the bulk of the mid-IR extragalactic emission comes from star formation and that the luminous galaxies seen by ISOCAM in the deep surveys are essentially starbursts obscured by dust. Nevertheless, this result does not exclude the possibility that the majority of the galaxies in the Universe have both AGN and starforming contributions. The results obtained by Elbaz et al. (2002) about the origin of the infrared background light based on a set of observed correlations and by Flores et al. (1999) evaluating the mid-IR part of the star formation are not significantly affected by the AGN contribution to the midIR extragalactic light.

Acknowledgements. F. D. dedicates this work to the memory of his professor Giuliano Giuricin, recently deceased, who introduced him to the study of AGNs. F. D. acknowledges support from the network ISO SURVEY set up by the European Commission under contract ERBFNRXCT960068 or its TMR program. H. F. was supported by a grant of the "Académie de la science". We thank the referee D. M. Alexander for his careful reading of the manuscript, interesting comments and suggestions which greatly improved the paper. We are grateful to H. Aussel for providing us with his flux list before publication. We also thank M. Arnaud, I. Perez-Fournon and F. La Franca for fruitful discussions and interesting suggestions.

\section{References}

Alexander, D. M., La Franca, F., Fiore, F., et al. 2001, ApJ, 554,18

Alexander, D. M., Brandt, W. N., Hornschemeier, A. E., et al. 2001a, AJ, 122, 2156

Almaini, O., Lawrence, A., \& Boyle, B. J. 1999, MNRAS, 305, 59

Altieri, B., Metcalfe, L., Kneib, J. P., et al. 1999, A\&A, 343, L65

Aussel, H., Cesarsky, C. J., Elbaz, D., \& Starck, J. L. 1999, A\&A, 342, 313

Aussel, H., et al. 2002, in preparation

Barger, A. L., Cowie, L. L., Trentham, N., et al. 1999, AJ, 117, 102

Brandt, W. N., Fabian, A. C., Takahashi, K., et al. 1997, MNRAS, 290, 617

Brandt, W. N., Alexander, D. M., Hornschemeier, A. E., et al. 2001a, AJ, in press [astro-ph/0108404]

Brandt, W. N., Hornschemeier, A. E., Alexander, D. M., et al. 2001, AJ, 122, 1

Cappi, M., Persic, M., Bassani, L., et al. 1999, A\&A, 350, 777

Carroll, S. M., Press, W. H., \& Turner, E. L. 1992, ARA\&A, 30, 499

Charmandaris, V., Laurent, O., Mirabel, I. F., et al. 1999, Ap\&SS, 266, 99

Chary, R., \& Elbaz, D. 2001, ApJ, 556, 562

Chen, L.-W., Fabian, A. C., \& Gendreau, K. C. 1997, MNRAS, 285,449

Clavel, J., Schulz, B., Altieri, B., et al. 2000, A\&A, 357, 839

Cohen, J. G., Cowie, L. L., Hogg, D. W., et al. 1996, ApJ, 471, L5

Cohen, J. G., Hogg, D. W., Blandford, R., et al. 2000, ApJ, 538, 29

Cohen, J. G. 2001, AJ, 121, 2895

Comastri, A., Setti, G., Zamorani, G., \& Hasinger, G. 1995, A\&A, 296, 1

Dawson, S., Stern, D., Bunker, A. J., et al. 2001, AJ, 122, 598

Elbaz, D., Cesarsky, C. J., Fadda, D., et al. 1999, A\&A, 351, L37

Elbaz, D., Cesarsky, C. J., Chanial, P., et al. 2002, A\&A, in press [astro-ph/0201328]

Fabian, A. C., \& Iwasawa, K. 1999, MNRAS, 303, L34

Fadda, D., Elbaz, D., Duc, P.-A., et al. 2000, A\&A

Fadda, D., et al. 2002, in preparation

Fioc, M., \& Rocca-Volmerange, B. 1997, A\&A, 326, 950

Flores, H., Hammer, F., Thuan, T. X., et al. 1999, ApJ, 517, 148

Flores, H., et al. 2002, in preparation

Franceschini, A., Aussel, H., Cesarsky, C., et al. 2001, A\&A, 378,1

Franceschini, A., Fadda, D., Flores, H., et al. 2001, ApJL, in press [astro-ph/01111413]

Gehrels, N. 1986, ApJ, 303, 336

Genzel, R., Lutz, D., Sturm, E., et al. 1998, ApJ, 498, 579

Guainazzi, M., Perola, G. C., Matt, G., et al. 1999, A\&A, 346, 407

Haas, M., Müller, S. A. H., Chini, R., et al. 2000, A\&A, 354, 453

Hasinger, G., Burg, F., Giacconi, R., et al. 1993, A\&A, 275, 1

Hasinger, G., Burg, F., Giacconi, R., et al. 1998, A\&A, 329, 482 
Hasinger, G., Altieri, B., Arnaud, M., et al. 2001, A\&A, 365, L45

Hauser, M. G., Arendt, R. G., Kelsall, T., et al. 1998, ApJ, 508,25

Hogg, D. W., Cohen, J. G., Blandford, R., et al. 1997, AJ, 115, 1418

Hogg, D. W., Pahre, M. A., Adelberger, K. L., et al. 2000, ApJ, 127,1

Hornschemeier, A. E., Brandt, W. N., Garminer, G. P., et al. 2001, AJ, 554, 742

Iwasawa, K., Matt, G., Guainazzi, M., \& Fabian, A. C. 2001, MNRAS, 326, 894

Laurent, O., Mirabel, I. F., Charmandaris, V., et al. 2000, A\&A, 359, 887

Lawson, A. J., \& Turner, J. L. 1997, MNRAS, 288, 920

Lehmann, I., Hasinger, G., Schmidt, M., et al. 2000, A\&A, 354, 35

Lehmann, I., Hasinger, G., Schmidt, M., et al. 2001, A\&A, 371, 833

Lowenthal, J., Koo, D. C., Guzmán, R., et al. 1997, ApJ, 481, 673

Lutz, D., Spoon, H. W. W., Rigopoulou, D., et al. 1998, ApJ, 505, L103

Madau, P., Ferguson, H. C., Dickinson, M. E., et al. 1996, MNRAS, 283, 1388

Matt, G., Guainazzi, M., Frontera, F., et al. 1997, A\&A, 325, L13

Matt, G., Guainazzi, M., Maiolino, R., et al. 1999, A\&A, 39, L42

Matt, G., Guainazzi, M., Maiolino, R., et al. 1999a, A\&A, 341, L39

Matt, G., Fabian, A. C., Guainazzi, M., et al. 2000, MNRAS, 318,173
Mushotzky, R. F., Cowie, L. L., Barge, A. J., \& Arnaud, K. A. 2000, Nature, 404, 459

Perola, G. C., Matt, G., Fiore, F., et al. 2000, A\&A, 358, 117

Pierre, M., Lidman, C., Hunstead, R., et al. 2001, A\&A, 372, L45

Puget, J.-L., Abergel, A., Bernard, J.-P., et al. 1996, A\&A, 308, L5

Richards, E. A., Kellermann, K. I., Fomalont, E. B., et al. 1998, AJ, 116, 1039

Richards, E. A. 2000, ApJ, 533, 611

Rowan-Robinson, M., Mann, R. J., Oliver, S. J., et al. 1997, MNRAS, 289, 490

Sambruna, R. M., Netzer, H., Kaspi, S., et al. 2001, ApJ, 546, L13

Severgnini, P., Maiolino, R., Salvati, M., et al. 2000, A\&A, 360, 457

Siebenmorgen, F., Moorwood, A., Freudling, W., \& Käufl, H. U. 1997, A\&A, 325, 450

Starck, J.-L., Aussel, H., Elbaz, D., Fadda, D., \& Cesarsky, C. 1999, A\&AS, 138, 365

Sturm, E., Lutz, D., Genzel, R., et al. 1996, A\&A, 315, 133

Sturm, E., Lutz, D., Tran, D., et al. 2000, A\&A, 358, 481

Stanev, T., \& Franceschini, A. 1998, ApJ, 494, L159

Tran, Q. D., Lutz, D., \& Gentzel, R. 2001, ApJ, 522, 527

Ueda, Y., Takahashi, T., Ishisaki, Y., et al. 1999, ApJ, 524, L11

Vecchi, A., Molendi, S., Guainazzi, M., et al. 1999, A\&A, 349, L73

Vignati, P., Molendi, S., Matt, G., et al. 1999, A\&A, 349, L57

Waddington, I., Windhorst, R. A., Cohen, S. H., et al. 1999, ApJ, 526, L77

Xu, C. 2000, ApJ, 541, 134 